\title{
Nonlinear layered lattice model and generalized solitary waves in imperfectly bonded structures
}

\author{
Karima R. Khusnutdinova, ${ }^{1, *}$ Alexander M. Samsonov, ${ }^{2}$ and Alexey S. Zakharov ${ }^{1}$ \\ ${ }^{1}$ Department of Mathematical Sciences, Loughborough University, Loughborough LE11 3TU, United Kingdom \\ ${ }^{2}$ Ioffe Physico-Technical Institute of the Russian Academy of Sciences, St. Petersburg 194021, Russia
}

(Received 21 November 2008; published 13 May 2009)

\begin{abstract}
We study nonlinear waves in a two-layered imperfectly bonded structure using a nonlinear lattice model. The key element of the model is an anharmonic chain of oscillating dipoles, which can be viewed as a basic lattice analog of a one-dimensional macroscopic waveguide. Long nonlinear longitudinal waves in a layered lattice with a soft middle (or bonding) layer are governed by a system of coupled Boussinesq-type equations. For this system we find conservation laws and show that pure solitary waves, which exist in a single equation and can exist in the coupled system in the symmetric case, are structurally unstable and are replaced with generalized solitary waves.
\end{abstract}

DOI: 10.1103/PhysRevE.79.056606

PACS number(s): 05.45.Yv, 62.30.+d, 43.25.+y

\section{INTRODUCTION}

Lattice models, i.e., ordered (in particular periodic), discrete systems of interacting particles, are often used to study nonlinear dynamics of condensed matter (e.g., [1-4]). Typically, lattice models allow for a slowly varying field approximation (long-wave approximation), which links them with continuum theories, and establishes relations between physical characteristics of a lattice and measurable macroparameters of the continuum. The latter is currently of considerable interest in connection with the question of applicability of continuum theories at microscales and nanoscales.

In 1938 Frenkel and Kontorova (FK) [5] used a simple chain of particles interacting with the nearest neighbors in the presence of an external periodic potential to model the dynamics of dislocations in metals in the vicinity of the dislocation core. One- and two-dimensional FK-type models have found numerous applications in studies of dynamics of molecular crystals, polymer chains, hydrogen-bonded chains, etc. (see $[4,6]$ and references therein). In 1955 the FermiPasta-Ulam (FPU) [7] model appeared in connection with the studies of the thermal conductivity of solids. It was observed that, counter intuitively, in anharmonic chains of particles (with weak nonlinearity in interaction potentials) there was no equipartition of energy among the modes of the harmonic approximation. This study has later led to the concept of a soliton, a localized (exponentially decaying to infinity) remarkably stable traveling nonlinear wave of permanent form [8]. In 1967 Toda [9] showed that a chain of particles with exponential interaction potentials also has exact solitary wave solutions. A modified Toda lattice with an external linear elastic term has been used to model the dynamics of a laminated composite in [10], establishing that stable envelope solitons play a central role in the dynamics of such a chain instead of the Toda solitons. In [11] the Toda lattice has been related to a different composite, where layers were oriented across the direction of wave propagation, having shown a possibility of existence of solitary waves in such

\footnotetext{
*Corresponding author: FAX: +44 (0)1509 223969; k.khusnutdinova@lboro.ac.uk
}

laminated structures. The so-called "zig-zag" models have been introduced to describe the nonlinear dynamics of molecular chains in [12], having shown that a geometric nonlinearity can have a strong effect on the dynamics of a chain, alongside with the nonlinearity of the interaction potential (often called the physical nonlinearity). Coupled KleinGordon (cKG) chains and corresponding cKG equations were proposed as a model for long longitudinal waves in bilayers, where nonlinearity comes only from the bonding material, and were used to study a number of dynamic properties of such coupled systems, including solitary waves, modulational instability of nonlinear multiphase wave trains, and energy exchange between the components (see [13] and references therein).

Lattice models are also often used to study fracture (e.g., [14-23]). In such models, fracture is introduced as vanishing links between respective elements of the lattice. It was shown that many dynamic properties of fracture can be described using linear lattice models. The lattice approach to fracture problems has several advantages compared to the classical continuum theories described, for example, in [24]. In particular, it allows for more natural formulations of the fracture criterion and more effective numerical approaches to the dynamics of fracture, for example, such difficult aspects as crack branching, although developing suitable lattice models is a complex problem in its own right (see $[20,21]$ and references there). It also allows one to construct a number of exact analytical solutions, using the discrete Fourier transform and the discrete Wiener-Hopf technique, overcoming certain difficulties of the classical continuum fracture problems (e.g., $[19,22,23])$. Lattice modeling of nonlinear waves in a delaminated bilayer has been attempted in [25], having shown the sensitivity of nonlinear waves to such extended inhomogeneities, and having invited further studies of the scattering of nonlinear waves in delaminated layered elastic waveguides [26-28].

In many cases the behavior of solids is affected by the existence of "microstructure," which could be of various nature. Most obvious sources of microstructure are the existence of molecules in polymers, grains in granular materials, crystallites in polycrystals, heterogeneity of mechanical properties of composites, as well as some other irregularities of materials. Again, there are two main approaches to the 
modeling of microstructured media: continuum theories (see, for example, [29-32] ) and lattice theories (e.g., [1-3] and references therein).

In this paper, we use a lattice model to study nonlinear waves in layered elastic waveguides with some softer material between the layers (for example, a soft adhesive bonding). The key element of this model is a complex chain of oscillating dipoles earlier considered as a linear model in [33]. The latter is a natural generalization of the linear model in [2,34] and a nonlinear model in [35]. Introduction of such models was mainly motivated by applications to the description of thin films, although they can also be viewed as a two-dimensional "footprint" of some three-dimensional crystals (e.g., [3]). The model proposed in [35] has also found applications to the description of some quasi-onedimensional crystals [36]. Interest to lattice models has been recently renewed in connection with so-called lattice materials (e.g., [37]) and nanostructures (e.g., [38]).

A natural question arising in connection with any lattice model is an existence of a mapping between a nonlinear lattice and some nonlinear partial differential equations for the continuum (e.g., [39]). Considering a simplified model of two coupled one-dimensional FPU chains with the soft bonding between them, one may expect to get a system of coupled Boussinesq-type equations in the continuum approximation. However, it is important to derive this system explicitly as an accurate nonlinear asymptotic model from a complex chain, which has all essential degrees of freedom of a real elastic waveguide, and takes into account both geometrical and physical sources of nonlinearity. This approach would be impossible until recently due to the large amount of required derivations. As shown in [40], derivations of this kind are now possible (although still difficult), using modern computer algebra packages (we used MATHEMATICA [41]).

Our paper is organized as follows. In Sec. II we consider the basic lattice waveguide and discuss the coincidence of the corresponding asymptotic continuum model describing long nonlinear longitudinal waves with the Boussinesq-type equation derived for a macroscopic waveguide using the nonlinear elasticity approach in [42]. The approach of this section can be applied to other lattice models used to study nonlinear waves or in molecular-dynamics simulations. We note that in the lattice waveguide under consideration, there is no simple analogy between long flexural and longitudinal waves. We finish the section with a possible extension of this basic model. Section III is devoted to a layered lattice model which we use to study nonlinear waves in two-layered waveguides with a layer of a softer material between them. Long nonlinear longitudinal waves in this lattice model are described by a system of coupled Boussinesq-type equations. We consider a version of coupled regularized Boussinesq(cRB)-type equations and find two nontrivial conservation laws, which are later used to control the accuracy of our numerical simulations. In Sec. IV we study nonlinear waves in a two-layered imperfectly bonded structure using the derived system. We show that the classical solitary wave solution, exponentially decaying to zero in its tail regions, which exists in the symmetric case, is structurally unstable. It is replaced with a generalized solitary wave having a copropagating oscillatory tail, which clearly manifests itself in

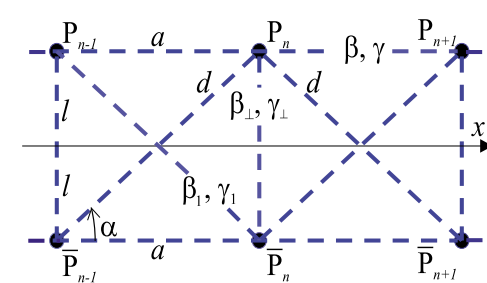

(a)

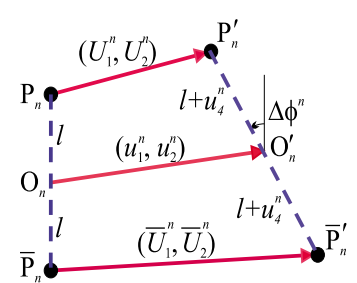

(b)
FIG. 1. (Color online) Dipole lattice model: an anharmonic chain of oscillating dipoles $\left(P_{n}, \bar{P}_{n}\right)$ described by four variables $\left(u_{1}^{n}, u_{2}^{n}, \Delta \phi^{n}, u_{4}^{n}\right)$.

numerical simulations. We theoretically predict the period and wavelength of these long-wave ripples, accompanying the core solitary wave, and compare them with numerical results. We conclude in Sec. V by discussing a possible link between the difference in the nature of nonlinear waves in layered structures with different types of bonding and recent experimental results in [27].

\section{DIPOLE LATTICE MODEL}

Our basic dipole lattice model consists of coupled FPUtype chains of interacting particles of mass $m$ shown in Fig. 1, which is viewed as an anharmonic chain of oscillating dipoles $\left(P_{n}, \bar{P}_{n}\right)$.

Each dipole has four degrees of freedom, and its displacements are described by the following variables: horizontal $\left(u_{1}^{n}\right)$ and vertical $\left(u_{2}^{n}\right)$ displacements of the geometrical center $O_{n}$, in-plane rotation of the dipole axis (to an angle $\Delta \varphi^{n}$ ), and change in a distance between the poles $\left(2 u_{4}^{n}\right)$. This is a nonlinear symmetric version of the linear model considered in connection with the dynamics of thin films and elastic trusses in [33]. The model generalizes the simpler chain of dipoles (with fixed distance between the poles) studied as a linear model in [2] (see also [3]) and [34] and as a nonlinear model in $[35]$.

\section{A. Governing equations}

The displacements of poles are given in terms of the dipole coordinates (see Fig. 1) as follows:

$$
\begin{gathered}
U_{1}^{n}=u_{1}^{n}-\left(l+u_{4}^{n}\right) \sin \Delta \varphi^{n}, \\
U_{2}^{n}=u_{2}^{n}+\left(l+u_{4}^{n}\right) \cos \Delta \varphi^{n}-l, \\
\bar{U}_{1}^{n}=u_{1}^{n}+\left(l+u_{4}^{n}\right) \sin \Delta \varphi^{n}, \\
\bar{U}_{2}^{n}=u_{2}^{n}-\left(l+u_{4}^{n}\right) \cos \Delta \varphi^{n}+l .
\end{gathered}
$$

Introducing $u_{3}^{n}=l \Delta \phi^{n}$ and assuming that rotations are small $\Delta \varphi^{n}=u_{3}^{n} / l \ll 1$, one can use truncated Taylor expansions in order to derive equations of motion up to quadratic terms.

The kinetic energy of the $n$th dipole has the form 


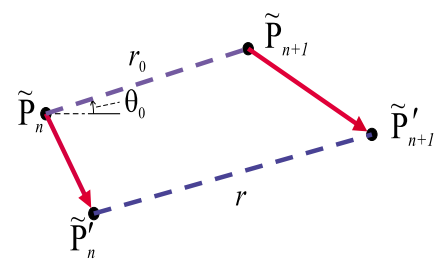

FIG. 2. (Color online) Distances between $\widetilde{P}_{n}$ and $\widetilde{P}_{n+1}$ and $\widetilde{P}_{n}^{\prime}$ and $\widetilde{P}_{n+1}$.

$$
T_{n}=\frac{M}{2}\left[\left(\dot{u}_{1}^{n}\right)^{2}+\left(\dot{u}_{2}^{n}\right)^{2}+\left(\dot{u}_{4}^{n}\right)^{2}+\left(1+\frac{u_{4}^{n}}{l}\right)^{2}\left(\dot{u}_{3}^{n}\right)^{2}\right],
$$

where $M=2 m$ is the dipole mass, and dot denotes derivative with respect to time. The potential energy of the $n$th dipole is defined by pairwise interactions between neighboring particles,

$$
\begin{aligned}
\Phi_{n}= & \Phi_{n, n+1}+\Phi_{\bar{n}, \overline{n+1}}+\Phi_{n, \overline{n+1}}+\Phi_{\bar{n}, n+1}+\Phi_{n-1, n}+\Phi_{\overline{n-1}, \bar{n}} \\
& +\Phi_{n-1, \bar{n}}+\Phi_{\overline{n-1}, n}+\Phi_{\perp},
\end{aligned}
$$

where overlines denote particles in the second ("bottom") row. The potential energy of interaction between any two neighboring particles is assumed to have the form

$$
\Phi_{*}\left(\Delta r_{*}\right)=\frac{\tilde{\beta}}{2} \Delta r_{*}^{2}+\frac{\tilde{\gamma}}{3} \Delta r_{*}^{3}+\ldots,
$$

where $\Delta r_{*}$ is the change in a distance between the particles, and $(\widetilde{\beta}, \widetilde{\gamma})$ denotes one of three possible pairs of interaction constants shown in Fig. 1. The change in a distance between any pole of the $n$th dipole $\left(\widetilde{P}_{n}\right)$ and any pole of the $(n+1)$ th dipole $\left(\widetilde{P}_{n+1}\right)$ is given by (see Fig. 2$)$

$$
\begin{aligned}
\Delta r_{\tilde{n}, n+1}= & {\left[\left(\tilde{U}_{1}^{n+1}-\tilde{U}_{1}^{n}+r_{0} \cos \theta_{0}\right)^{2}\right.} \\
& \left.+\left(\tilde{U}_{2}^{n+1}-\tilde{U}_{2}^{n}+r_{0} \sin \theta_{0}\right)^{2}\right]^{1 / 2}-r_{0},
\end{aligned}
$$

where $\tilde{U}_{1}^{n}, \tilde{U}_{2}^{n}$ denote horizontal and vertical displacements of the pole $\widetilde{P}_{n}$. The change in a distance between the poles of the $n$th dipole is given by $\Delta r_{\perp}=2 u_{4}^{n}$.

Introducing the differences $\Delta x=\widetilde{U}_{1}^{n+1}-\widetilde{U}_{1}^{n}, \Delta y=\widetilde{U}_{2}^{n+1}-\tilde{U}_{2}^{n}$, and assuming that these differences are small compared to $r_{0}$, i.e., $\Delta x / r_{0} \ll 1, \Delta y / r_{0} \ll 1$, one can use expansions

$$
\begin{aligned}
\Delta r_{\tilde{n}, \tilde{n+1}}= & \Delta x \cos \theta_{0}+\Delta y \sin \theta_{0}+\frac{1}{2 r_{0}}\left(\Delta x \sin \theta_{0}\right. \\
& \left.+\Delta y \cos \theta_{0}\right)^{2}-\frac{1}{2 r_{0}^{2}}\left(\Delta x \cos \theta_{0}+\Delta y \sin \theta_{0}\right) \\
& \times\left(\Delta x \sin \theta_{0}+\Delta y \cos \theta_{0}\right)^{2}+\ldots
\end{aligned}
$$

Substitution of Eqs. (1) and (2) into the Euler-Lagrange equations

$$
\frac{d}{d t}\left(\frac{\partial T_{n}}{\partial \dot{u}_{i}^{n}}\right)-\frac{\partial T_{n}}{\partial u_{i}^{n}}+\frac{\partial \Phi_{n}}{\partial u_{i}^{n}}=0
$$

yields four difference-differential equations

$$
\begin{gathered}
M \ddot{u}_{1}^{n}+\frac{\partial \Phi_{n}}{\partial u_{1}^{n}}=0, \quad M \ddot{u}_{2}^{n}+\frac{\partial \Phi_{n}}{\partial u_{2}^{n}}=0, \\
M\left[\ddot{u}_{3}^{n}\left(1+\frac{u_{4}^{n}}{l}\right)^{2}+2 \frac{\dot{u}_{3}^{n} \dot{u}_{4}^{n}}{l}\left(1+\frac{u_{4}^{n}}{l}\right)\right]+\frac{\partial \Phi_{n}}{\partial u_{3}^{n}}=0, \\
M\left[\ddot{u}_{4}^{n}-\frac{\left(\dot{u}_{3}^{n}\right)^{2}}{l}\left(1+\frac{u_{4}^{n}}{l}\right)\right]+\frac{\partial \Phi_{n}}{\partial u_{4}^{n}}=0,
\end{gathered}
$$

where

$$
\begin{aligned}
-\frac{\partial \Phi_{n}}{\partial u_{i}^{n}}= & b_{i j} \Delta_{j}^{n}+c_{i j}\left(\Delta^{2}\right)_{j}^{n}+d_{i j} u_{j}^{n}+g_{i j k}\left(\Delta^{2}\right)_{j}^{n} \Delta_{k}^{n} \\
& +h_{i j k}\left[\left(\Delta^{2}\right)_{j}^{n}\left(\Delta^{2}\right)_{k}^{n}+\Delta_{j}^{n} \Delta_{k}^{n}\right]+\left(l_{i j} u_{3}^{n}+\widetilde{l}_{i j} u_{4}^{n}\right)\left(\Delta^{2}\right)_{j}^{n} \\
& +\left(m_{i j} u_{3}^{n}+\tilde{m}_{i j} u_{4}^{n}\right) \Delta_{j}^{n}+p_{i j k} u_{j}^{n} u_{k}^{n},
\end{aligned}
$$

and $\Delta_{j}^{n}=u_{j}^{n+1}-u_{j}^{n-1},\left(\Delta^{2}\right)_{j}^{n}=u_{j}^{n+1}-2 u_{j}^{n}+u_{j}^{n-1}$. (We assume the summation with respect to repeated indices.) The coefficients of Eq. (6) are given in Appendix A.

\section{B. Models for long longitudinal and flexural waves}

To study long waves, one can use the continuum approximation of the governing Eqs. (5) (e.g., [32]),

$$
u_{i}^{n}(t)=u_{i}(x, t) \text {, }
$$

$$
\begin{aligned}
u_{i}(x \pm a, t)= & u_{i}(x, t) \pm a u_{i_{x}}(x, t)+\frac{a^{2}}{2} u_{i_{x x}}(x, t) \pm \frac{a^{3}}{6} u_{i_{x x x}}(x, t) \\
& +\frac{a^{4}}{24} u_{i_{x x x}}(x, t)+\ldots,
\end{aligned}
$$

and subsequent asymptotic analysis of the arising system of nonlinear PDEs,

$$
\begin{gathered}
M u_{1_{t t}}=2 b_{14} a\left(u_{4_{x}}+\frac{a^{2}}{6} u_{4_{x x x}}\right)+c_{11} a^{2}\left(u_{1_{x x}}+\frac{a^{2}}{12} u_{1_{x x x x}}\right)+N_{1}, \\
M u_{2_{t t}}=-2 b_{14} a\left(u_{3_{x}}+\frac{a^{2}}{6} u_{3_{x x x}}\right)+c_{22} a^{2}\left(u_{2_{x x}}+\frac{a^{2}}{12} u_{2_{x x x x}}\right)+N_{2}, \\
M\left[u_{3_{t t}}\left(1+\frac{u_{4}}{l}\right)^{2}+2 \frac{u_{3_{t}} u_{4_{t}}}{l}\left(1+\frac{u_{4}}{l}\right)\right] \\
=2 b_{14} a\left(u_{2_{x}}+\frac{a^{2}}{6} u_{2_{x x x}}\right)+c_{33} a^{2}\left(u_{3_{x x}}+\frac{a^{2}}{12} u_{3_{x x x x}}\right) \\
+d_{33} u_{3}+N_{3}, \\
M\left[\begin{array}{c}
\left.u_{4_{t t}}-\frac{\left(u_{3}\right)^{2}}{l}\left(1+\frac{u_{4}}{l}\right)\right] \\
=-2 b_{14} a\left(u_{1_{x}}+\frac{a^{2}}{6} u_{1_{x x x}}\right)-c_{22} a^{2}\left(u_{4_{x x}}+\frac{a^{2}}{12} u_{4_{x x x x}}\right)
\end{array}\right. \\
+d_{44} u_{4}+N_{4} .
\end{gathered}
$$


The truncated nonlinear terms $N_{1-4}$ in the right-hand side of Eqs. (7) are given in Appendix B.

The derivation of the asymptotic long-wave models for predominantly longitudinal or flexural waves is based on an observation that in the linear approximation the system (7) uncouples into two subsystems, describing the interaction between longitudinal and dipole (or axial) oscillations, and transversal and rotational oscillations [33]. Introducing dimensionless variables

$$
\tilde{u}_{i}=\frac{u_{i}}{A_{i}}, \quad \tilde{t}=\frac{t}{T}, \quad \tilde{x}=\frac{x}{X},
$$

looking for predominantly longitudinal waves, and denoting $X / T=v=\mathcal{O}(1)$, one can find the scaling

$$
\frac{A_{1}}{a} \sim \varepsilon^{1 / 2}, \quad \frac{A_{2}}{a} \sim \varepsilon^{3 / 2}, \quad \frac{A_{3}}{a} \sim \varepsilon^{2}, \quad \frac{A_{4}}{a} \sim \varepsilon, \quad \frac{a}{X} \sim \varepsilon^{1 / 2},
$$

when the leading-order behavior of the system is described by the subsystem of equations for $\tilde{u}_{1}$ and $\tilde{u}_{4}$,

$$
\begin{aligned}
& M v^{2} \tilde{u}_{1_{\tilde{t}}}-a^{2}\left(2 b_{14} \tilde{u}_{4_{\tilde{x}}}+c_{11} \tilde{u}_{\tilde{T}_{\widetilde{x x}}}\right)+\varepsilon a^{3}\left(2 m_{41} \tilde{u}_{4} \tilde{u}_{4_{\tilde{x}}}+8 h_{123} \tilde{u}_{1_{\tilde{x}}} \tilde{u}_{4_{\tilde{x}}}\right. \\
& \left.+l_{12} \tilde{u}_{4} \tilde{u}_{1_{\widetilde{x x}}}-2 g_{111} \tilde{u}_{1_{\tilde{x}}} \tilde{u}_{1_{\widetilde{x x}}}\right)-\varepsilon a^{2}\left(\frac{b_{14}}{3} \tilde{u}_{4_{\widehat{x x x}}}+\frac{c_{11}}{12} \tilde{u}_{1_{\widetilde{x x x x}}}\right) \\
& +\mathcal{O}\left(\varepsilon^{2}\right)=0 \\
& 2 b_{14} \tilde{u}_{1_{\tilde{x}}}-d_{44} \tilde{u}_{4}+\varepsilon\left[\frac{M v^{2}}{a^{2}} \tilde{u}_{4_{\tilde{t t}}}+c_{22} \tilde{u}_{4_{\tilde{x} x}}+\frac{b_{14}}{3} \tilde{u}_{1_{\widetilde{x x}}}\right. \\
& -a\left(p_{444} \widetilde{u}_{4}^{2}+2 m_{41} \tilde{u}_{4} \tilde{u}_{\tilde{x}_{\tilde{x}}}+4 h_{123}\left(\widetilde{u}_{1_{\tilde{x}}}\right)^{2}\right]+\mathcal{O}\left(\varepsilon^{2}\right)=0 .
\end{aligned}
$$

Here $m_{41}=-m_{14}=m_{24}+\frac{4 \beta_{1}}{d} \cos \alpha$. From Eq. (11), $\tilde{u}_{4}$ can be expressed in terms of $\tilde{u}_{1}$ by asymptotic reiteration,

$$
\begin{aligned}
\tilde{u}_{4}= & \frac{2 b_{14}}{d_{44}} \tilde{u}_{1_{\tilde{x}}}+\varepsilon\left[4 a\left(\frac{b_{14} m_{41}}{d_{44}^{2}}-\frac{h_{123}}{d_{44}}-\frac{b_{14}^{2} p_{444}}{d_{44}^{3}}\right)\left(\tilde{u}_{1_{\tilde{x}}}\right)^{2}\right. \\
& \left.+\frac{2 b_{14} M v^{2}}{a^{2} d_{44}^{2}} \tilde{u}_{1_{\tilde{t} \tilde{x}}}+\left(\frac{2 b_{14} c_{22}}{d_{44}^{2}}+\frac{b_{14}}{3 d_{44}}\right) \tilde{u}_{1_{\widetilde{x x x}}}\right]+\mathcal{O}\left(\varepsilon^{2}\right) .
\end{aligned}
$$

Substituting Eq. (12) into Eq. (10), returning to the original dimensional variables and denoting $u_{1}=u$ one obtains to $\mathcal{O}(\varepsilon)$, the equation for long nonlinear longitudinal waves in the form

$$
u_{t t}-c_{0}^{2} u_{x x}=E_{1} u_{x} u_{x x}+E_{2} u_{t t x x}+E_{3} u_{x x x x}
$$

where the coefficients $c_{0}, E_{1}, E_{2}$, and $E_{3}$ are expressed in terms of the lattice parameters as follows:

$$
c_{0}^{2}=\frac{a^{2}}{M}\left(2 \beta+\beta_{\perp} \nu\right), \quad \nu=\frac{2 \beta_{1} \cos ^{2} \alpha}{\beta_{\perp}+2 \beta_{1} \sin ^{2} \alpha},
$$

$$
\begin{gathered}
E_{1}=\frac{a^{2}}{M}\left\{3 \beta_{\perp} \nu(\nu+1)^{2} \sin ^{2} \alpha+a\left[4 \gamma-2 \gamma_{\perp} \nu^{3} \tan ^{3} \alpha\right.\right. \\
\left.\left.+\frac{\gamma_{1}}{2}\left(\frac{\beta_{\perp} \nu}{\beta_{1} \cos \alpha}\right)^{3}\right]\right\}, \quad E_{2}=l^{2} \nu^{2}, \\
E_{3}=\frac{a^{4}}{12 M}\left[2 \beta+\beta_{\perp} \nu\left(1-3 \nu \tan ^{2} \alpha\right)\right] .
\end{gathered}
$$

Equation (13) derived here as an asymptotic approximation of the initial dipole lattice model coincides with the so-called doubly dispersive equation (DDE), earlier derived for a macroscopic waveguide using the nonlinear elasticity approach [42-44]. A similar equation has also appeared in the study of waves in microstructured solids (see $[45,46]$ and references therein).

For the motions under consideration, the equation above is asymptotically equivalent to both the integrable by the inverse scattering transform (IST) nonlinear string equation $[47,48]$,

$$
u_{t t}-c_{0}^{2} u_{x x}=E_{1} u_{x} u_{x x}+E_{23} u_{x x x x},
$$

where $E_{23}=E_{2} c_{0}^{2}+E_{3}$, and the regularized Boussinesq equation,

$$
u_{t t}-c_{0}^{2} u_{x x}=E_{1} u_{x} u_{x x}+\widetilde{E}_{23} u_{t t x x},
$$

where $\tilde{E}_{23}=E_{23} / c_{0}^{2}$. The latter is nonintegrable by the IST, however, is preferable from the viewpoint of numerical simulations due to suppression of the short wave instability (e.g., see $[49,50])$.

To leading order, the relation between the axial displacement and the horizontal strain is given by $u_{4}=-l \nu u_{1_{x}}+\ldots$ which can be viewed as a lattice analog of Poisson's effect in elasticity [51], with parameter $\nu$ corresponding to Poisson's ratio, while higher-order terms in Eq. (12) provide corrections to this relation. Weak (i.e., higher order in $\varepsilon$ ) transversal and rotational motions are slaved to longitudinal waves (see Appendix C).

It is interesting to compare the previous case of the dominant longitudinal waves with the case of the dominant flexural waves by introducing a different scaling,

$$
\begin{gathered}
\frac{A_{1}}{a} \sim \varepsilon^{3 / 2}, \quad \frac{A_{2}}{a} \sim \varepsilon^{1 / 2}, \quad \frac{A_{3}}{a} \sim \varepsilon, \quad \frac{A_{4}}{a} \sim \varepsilon^{2}, \\
\frac{a}{X} \sim \varepsilon^{1 / 2}, \quad \frac{X}{T}=v \sim 1 .
\end{gathered}
$$

Up to $\mathcal{O}\left(\varepsilon^{2}\right)$ this yields the following subsystem of equations for $\widetilde{u}_{2}$ and $\tilde{u}_{3}$ :

$$
\begin{gathered}
\tilde{u}_{2_{\tilde{t}}}+\frac{a^{2}}{M v^{2}}\left[\left(2 b_{14} \tilde{u}_{3_{\tilde{x}}}-c_{22} \tilde{u}_{2_{\widetilde{x x}}}\right)+\varepsilon\left(\frac{b_{14}}{3} \tilde{u}_{3_{\widetilde{x x x}}}-\frac{c_{22}}{12} \tilde{u}_{\tilde{x}_{\widetilde{x x x} x}}\right)\right]=0, \\
\tilde{u}_{2_{\tilde{x}}}+\frac{d_{33}}{2 b_{14}} \tilde{u}_{3}+\frac{\varepsilon}{2 b_{14}}\left(-\frac{M v^{2}}{a^{2}} \tilde{u}_{3_{\tilde{t t}}}+c_{33} \tilde{u}_{3_{\widetilde{x x}}}+\frac{b_{14}}{3} \tilde{u}_{2_{\widetilde{x} x x}}\right)=0 .
\end{gathered}
$$

Eliminating $\tilde{u}_{3}$ from the second equation by asymptotic reiteration (to leading order $\tilde{u}_{3}=l \tilde{u}_{2 \widetilde{x}}+\ldots$ ) and returning to the 


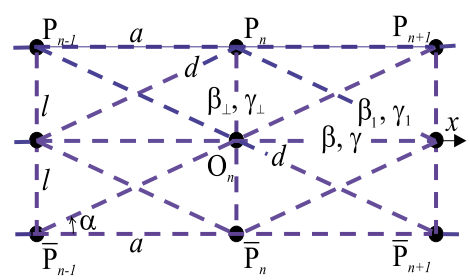

(a)

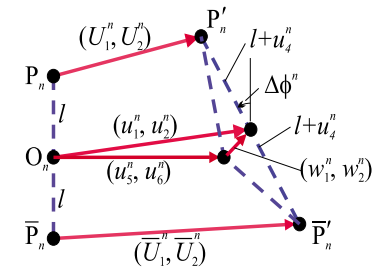

(b)

FIG. 3. (Color online) Extension of the dipole lattice model: two welded identical dipole lattices; variables $u_{1-4}^{n}$ define displacements of the boundary particles $P_{n}$ and $\widetilde{P}_{n}$, variables $u_{5-6}^{n}$ correspond to the displacements of the central particles $O_{n}$.

original variables, one arrives at the linear equation for $u_{2}$

$$
u_{2_{t t}}=l^{2} u_{2_{t t x x}}-\frac{2 a^{2} l^{2}}{M} \beta u_{2_{x x x x}},
$$

which is an analog of the equation for flexural waves in elastic waveguides, when the effect of rotational inertia is taken into account, but shear deformations are neglected (e.g., [52]). Thus, there is no simple analogy between long nonlinear longitudinal and flexural waves-at least-within the scope of our model.

\section{Extension of the dipole lattice model}

A natural extension of the basic dipole lattice model consists in considering a layered lattice with perfect or ideal interface (e.g., [53]), where two identical lattice waveguides are welded together (Fig. 3). Variables $u_{1-4}^{n}$ corresponding to the displacements of the boundary particles $P_{n}$ and $\bar{P}_{n}$ are defined as above. The horizontal and vertical displacements of the central particles $O_{n}$ are denoted by variables $u_{5}^{n}$ and $u_{6}^{n}$, respectively (see Fig. 3).

Deriving discrete and continuous equations as above and introducing dimensionless variables (8), one can find the predominantly longitudinal waves scaling in the form

$$
\begin{gathered}
\frac{A_{1}}{a} \sim \frac{A_{5}}{a} \sim \varepsilon^{1 / 2}, \quad \frac{A_{2}}{a} \sim \frac{A_{6}}{a} \sim \varepsilon^{3 / 2}, \quad \frac{A_{3}}{a} \sim \varepsilon^{2}, \\
\frac{A_{4}}{a} \sim \varepsilon, \quad \frac{a}{X} \sim \varepsilon^{1 / 2}, \quad \frac{X}{T}=v \sim 1 .
\end{gathered}
$$

Assuming that central particles do not displace too far from the geometrical center of the dipole, we write

$$
\tilde{u}_{1}=\tilde{u}_{5}+\varepsilon \tilde{w}_{1}+\ldots, \quad \tilde{u}_{2}=\tilde{u}_{6}+\varepsilon \tilde{w}_{2}+\ldots,
$$

where $\widetilde{w}_{1,2}$ are unknown functions. This assumption is an analog of the near planar cross-section hypothesis in the theory of elastic rods and bars (see $[44,45]$ ). One can asymptotically derive an independent subsystem of equations for predominantly longitudinal waves and, consequently, an equation for $u_{5}$ or $u_{1}$ (see Appendix D). The latter in dimensional variables has the form

$$
u_{1_{t t}}-c_{0}^{2} u_{1_{x x}}=G_{1} u_{1_{x}} u_{1_{x x}}+G_{2} u_{1_{t t x x}}+G_{3} u_{1_{x x x x}}+G_{4} u_{1_{t t t t}},
$$

where

$$
\begin{aligned}
& c_{0}^{2}= \frac{a^{2}}{M}\left(3 \beta+2 \beta_{\perp} \nu\right), \quad \nu=\frac{2 \beta_{1} \cos ^{2} \alpha}{\beta_{\perp}+2 \beta_{1} \sin ^{2} \alpha}, \\
& G_{1}= \frac{a^{2}}{M}\left\{6 \beta_{\perp} \nu(1+\nu)^{2} \sin ^{2} \alpha\right. \\
&+\left.a\left[6 \gamma-4 \gamma_{\perp} \nu^{3} \tan ^{3} \alpha+\gamma_{1}\left(\frac{\beta_{\perp} \nu}{\beta_{1} \cos \alpha}\right)^{3}\right]\right\}, \\
& G_{2}=\frac{1}{3}\left[2 l^{2} \nu^{2}-a^{2}\left(\frac{1}{2}-\frac{\beta+\beta_{\perp} \nu}{\beta_{1} \cos ^{2} \alpha}\right)\right], \\
& G_{3}= \frac{a^{4}}{M}\left(\frac{3}{4} \beta-\frac{1}{3} \beta_{\perp} \nu-\frac{\beta^{2}}{2 \beta_{1} \cos ^{2} \alpha}-\frac{\beta \beta_{\perp} \nu}{\beta_{1} \cos ^{2} \alpha}\right), \\
& G_{4}=-\frac{M}{18 \beta_{1} \cos ^{2} \alpha},
\end{aligned}
$$

and $u_{4}=-l \nu u_{1}^{\prime}+\ldots$. Note that this is also a Boussinesq-type equation, but unlike (13) it contains a fourth order time derivative. However, for this regime the Eq. (18) is again asymptotically equivalent to both (15) and (16) (with the refined coefficients). Similar to previous section, for this asymptotic regime weak transversal and rotational motions are slaved to the longitudinal waves.

The dominant flexural waves can be considered by introducing the scaling

$$
\begin{gathered}
\frac{A_{1}}{a} \sim \frac{A_{5}}{a} \sim \varepsilon^{3 / 2}, \quad \frac{A_{2}}{a} \sim \frac{A_{6}}{a} \sim \varepsilon^{1 / 2}, \quad \frac{A_{3}}{a} \sim \varepsilon, \\
\frac{A_{4}}{a} \sim \varepsilon^{2}, \quad \frac{a}{X} \sim \varepsilon^{1 / 2}, \quad \frac{X}{T}=v \sim 1 .
\end{gathered}
$$

Repeating the same steps as for longitudinal waves, one arrives at a more accurate equation for flexural waves compared to a single-layer lattice,

$$
\begin{aligned}
u_{2_{t t}}= & \frac{2}{3} l^{2}\left(1-\frac{\nu}{4}\right) u_{2_{t t x x}}-\frac{2 a^{2} l^{2}}{3 M}\left(3 \beta+\frac{3}{4} \beta_{\perp} \nu\right) u_{2_{x x x x}} \\
& -\frac{\nu M}{18 \beta_{1} \cos ^{2} \alpha} u_{2_{t t t t}}
\end{aligned}
$$

This is an analog of the equation for the linear flexural waves in an elastic waveguide, when the deflection due to shear is taken into account (e.g., [52]). The relation between $u_{2}$ and $u_{6}$ and an equation for $u_{6}$ is given in Appendix E.

\section{LAYERED LATTICE MODEL}

Motivated by our studies of longitudinal bulk nonlinear waves in layered waveguides [26-28], we consider two waveguides with a layer of a softer material between them 


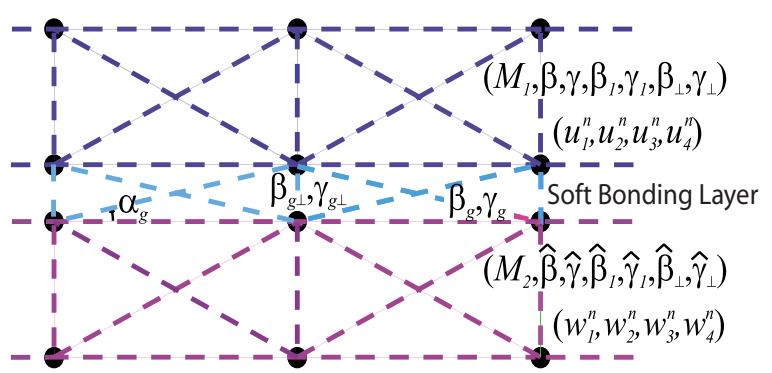

FIG. 4. (Color online) Layered lattice model: two dipole lattices with an intermediate soft bonding layer.

(for example, a soft adhesive, usually modeled as an imperfect interface, e.g., [53,54]). Layered structures become increasingly important in physics (e.g., in microelectronics and aerospace engineering). We use a layered lattice model shown in Fig. 4 and discuss asymptotic models for long nonlinear longitudinal waves. The notations for lattice parameters and variables are shown in Fig. 4.

\section{A. Coupled Boussinesq-type equations}

To derive the Euler-Lagrange equations for the composite lattice, one has to form the total potential energy of the $n$th lattice element,

$$
\Phi_{n}^{\text {total }}=\Phi_{n}+\hat{\Phi}_{n}+\Phi_{n}^{g}
$$

where $\Phi_{n}$ is the potential energy of the $n$th dipole of the upper layer [given by formulas (2)-(4)], $\hat{\Phi}_{n}$ is given by similar formulas for the potential energy of the $n$th dipole of the lower layer, and $\Phi_{n}^{g}$ is the energy of interactions between the layers (i.e., energy of the bonding layer, or glue, for brevity),

$$
\Phi_{n}^{g}=\Phi_{\bar{n}, n+1}^{g}+\Phi_{n, \overline{n+1}}^{g}+\Phi_{n-1, n}^{g}+\Phi_{n-1, \bar{n}}^{g}+\Phi_{\perp}^{g} .
$$

Here, the relevant formulae are similar to Eqs. (2)-(4) but refer to particles interacting via the bonding layer.

We assume that the bonding layer is soft and introduce the scaling

$$
\beta_{g}=\varepsilon^{2} \widetilde{\beta}_{g}, \quad \beta_{g \perp}=\varepsilon^{2} \widetilde{\beta}_{g \perp}, \quad \gamma_{g}=\varepsilon^{2} \widetilde{\gamma}_{g}, \quad \gamma_{g \perp}=\varepsilon^{2} \widetilde{\gamma}_{g} .
$$

One can derive the governing equations, their continuum approximation, and then use the scaling from Sec. II for the case of predominantly longitudinal waves to asymptotically derive the subsystem of four equations for $\tilde{u}_{1}, \tilde{u}_{4}, \widetilde{w}_{1}$, and $\widetilde{w}_{4}$, which are similar to a pair of coupled single-layer subsystems,

$$
\begin{aligned}
& M_{1} v^{2} \tilde{u}_{\tilde{I}_{\tilde{t}}}-a^{2}\left(2 b_{14} \tilde{u}_{4_{\tilde{x}}}+c_{11} \tilde{u}_{1_{\widetilde{x x}}}\right)+\varepsilon a^{3}\left(2 m_{41} \tilde{u}_{4} \tilde{u}_{4_{\tilde{x}}}\right. \\
& \left.+8 h_{123} \tilde{u}_{1_{\tilde{x}}} \tilde{u}_{4_{\tilde{x}}}+l_{12} \tilde{u}_{4} \tilde{u}_{1_{\widetilde{x x}}}-2 g_{111} \tilde{u}_{1_{\tilde{x}}} \tilde{u}_{1_{\widetilde{x x}}}\right) \\
& -\varepsilon a^{2}\left[\frac{b_{14}}{3} \tilde{u}_{4_{\tilde{x x x}}}+\frac{c_{11}}{12} \tilde{u}_{1_{\tilde{x x x x}}}-2 \widetilde{\beta}_{g} \cos ^{2} \alpha_{g}\left(\tilde{u}_{1}-\widetilde{w}_{1}\right)\right] \\
& +\mathcal{O}\left(\varepsilon^{2}\right)=0 \text {, }
\end{aligned}
$$

$$
\begin{aligned}
& 2 b_{14} \tilde{u}_{1_{\tilde{x}}}-d_{44} \tilde{u}_{4}+\varepsilon\left[\frac{M_{1} v^{2}}{a^{2}} \tilde{u}_{4_{\tilde{t}}}+c_{22} \tilde{u}_{\tilde{x}_{\tilde{x x}}}+\frac{b_{14}}{3} \tilde{u}_{1_{\tilde{x} x \tilde{x}}}\right. \\
& \left.-a\left(p_{444} \tilde{u}_{4}^{2}+2 m_{41} \tilde{u}_{4} \tilde{u}_{1_{\tilde{x}}}+4 h_{123}\left(\tilde{u}_{1_{\tilde{x}}}\right)^{2}\right)\right]+\mathcal{O}\left(\varepsilon^{2}\right)=0, \\
& M_{2} v^{2} \widetilde{w}_{1_{\tilde{t t}}}-a^{2}\left(2 \hat{b}_{14} \widetilde{w}_{4_{\tilde{x}}}+\hat{c}_{11} \widetilde{w}_{1_{\widetilde{x x}}}\right)+\varepsilon a^{3}\left(2 \hat{m}_{41} \widetilde{w}_{4} \widetilde{w}_{4_{\tilde{x}}}\right. \\
& \left.+8 \hat{h}_{123} \widetilde{w}_{1_{\tilde{x}}} \widetilde{w}_{4_{\tilde{x}}}+\hat{l}_{12} \widetilde{w}_{4} \widetilde{w}_{1_{\widetilde{x}}}-2 \hat{g}_{111} \widetilde{w}_{1_{\tilde{x}}} \widetilde{w}_{1_{\widetilde{x x}}}\right) \\
& -\varepsilon a^{2}\left(\frac{\hat{b}_{14}}{3} \widetilde{w}_{4_{\tilde{x x x}}}+\frac{\hat{c}_{11}}{12} \widetilde{w}_{1_{\widetilde{x x x x}}}-2 \widetilde{\beta}_{g} \cos ^{2} \alpha_{g}\left(\widetilde{w}_{1}-\widetilde{u}_{1}\right)\right) \\
& +\mathcal{O}\left(\varepsilon^{2}\right)=0 \text {, } \\
& 2 \hat{b}_{14} \widetilde{w}_{1_{\tilde{x}}}-\hat{d}_{44} \widetilde{w}_{4}+\varepsilon\left[\frac{M_{2} v^{2}}{a^{2}} \widetilde{w}_{4_{\tilde{t} t}}+\hat{c}_{22} \widetilde{w}_{4_{\tilde{x x}}}+\frac{\hat{b}_{14}}{3} \widetilde{w}_{1_{\tilde{x x x}}}\right. \\
& \left.-a\left(\hat{p}_{444} \widetilde{w}_{4}^{2}+2 \hat{m}_{41} \widetilde{w}_{4} \widetilde{w}_{1_{\tilde{x}}}+4 \hat{h}_{123}\left(\widetilde{w}_{1_{\tilde{x}}}\right)^{2}\right)\right]+\mathcal{O}\left(\varepsilon^{2}\right)=0 .
\end{aligned}
$$

Eliminating $\tilde{u}_{4}$ and $\tilde{w}_{4}$ from the equations above, we can describe the leading-order behavior of the system in terms of $\tilde{u}_{1}$ and $\tilde{w}_{1}$ by coupled doubly dispersive equations (cDDE), which in the original dimensional variables have the form (we denote $u_{1}=u$ and $w_{1}=w$ )

$$
\begin{gathered}
u_{t t}-c_{0}^{2} u_{x x}=E_{1} u_{x} u_{x x}+E_{2} u_{t t x x}+E_{3} u_{x x x x}-E_{4}(u-w), \\
w_{t t}-\hat{c}_{0}^{2} w_{x x}=\hat{E}_{1} w_{x} w_{x x}+\hat{E}_{2} w_{t t x x}+\hat{E}_{3} w_{x x x x}-\hat{E}_{4}(w-u) .
\end{gathered}
$$

The coefficients $c_{0}, E_{1}, E_{2}$, and $E_{3}$ are given by formulas in (14), where $M \rightarrow M_{1}$, and $E_{4}=\frac{2 \beta_{g}}{M_{1}} \cos ^{2} \alpha_{g}$. Similar coefficients in the second equation are obtained by replacing the upper layer constants with that of the lower layer. Using leading-order relations $u_{x x}=u_{t t} / c_{0}^{2}+\ldots, w_{x x}=w_{t t} / \hat{c}_{0}^{2}+\ldots$, one can show that for the motions under consideration this system is asymptotically equivalent to different types of coupled Boussinesq equations. We will consider the form of cRB equations due to its advantages from the viewpoint of numerical simulations,

$$
\begin{gathered}
u_{t t}-c_{0}^{2} u_{x x}=E_{1} u_{x} u_{x x}+\left(E_{2}+\frac{E_{3}}{c_{0}^{2}}\right) u_{t t x x}-E_{4}(u-w), \\
w_{t t}-\hat{c}_{0}^{2} w_{x x}=\hat{E}_{1} w_{x} w_{x x}+\left(\hat{E}_{2}+\frac{\hat{E}_{3}}{\hat{c}_{0}^{2}}\right) w_{t t x x}-\hat{E}_{4}(w-u) .
\end{gathered}
$$

Let us note that relaxing conditions on the coefficients of the bonding layer,

$$
\beta_{g}=\varepsilon \widetilde{\beta}_{g}, \quad \beta_{g \perp}=\varepsilon \widetilde{\beta}_{g \perp}, \quad \gamma_{g}=\varepsilon \widetilde{\gamma}_{g}, \quad \gamma_{g \perp}=\varepsilon \widetilde{\gamma}_{g},
$$

and repeating the steps above, one can obtain a more general asymptotic long-wave model, which in dimensional variables has the form 


$$
\begin{aligned}
u_{t t}-\left(c_{0}^{2}+c_{g}^{2}\right) u_{x x}= & E_{1} u_{x} u_{x x}+E_{2} u_{t t x x}+E_{3} u_{x x x x} \\
& -\left(E_{4}+E_{5} w_{x}\right)(u-w)-E_{6} w_{x x}, \\
w_{t t}-\left(\hat{c}_{0}^{2}+\hat{c}_{g}^{2}\right) w_{x x}= & \hat{E}_{1} w_{x} w_{x x}+\hat{E}_{2} w_{t t x x}+\hat{E}_{3} w_{x x x x} \\
& -\left(\hat{E}_{4}+\hat{E}_{5} u_{x}\right)(w-u)-\hat{E}_{6} u_{x x},
\end{aligned}
$$

where, in addition to coefficients in Eqs. (20),

$$
\begin{gathered}
c_{g}^{2}=\frac{l^{2} \nu^{2}}{M_{1}}\left(\beta_{g \perp}+2 \beta_{g} \sin ^{2} \alpha_{g}\right), \\
E_{6}=\frac{a^{2}}{4 M_{1}}\left\{2 \beta _ { g } \left[2 \cos ^{2} \alpha_{g}+(\nu+\hat{\nu}) \tan \alpha \sin 2 \alpha_{g}\right.\right. \\
\left.\left.+\nu \hat{\nu} \tan ^{2} \alpha \sin ^{2} \alpha_{g}\right]+\beta_{g \perp} \nu \hat{\nu} \tan ^{2} \alpha\right\},
\end{gathered}
$$

while similar coefficients in the second equation are obtained by replacing the upper layer constants with that of the lower layer. System (20) is an asymptotic reduction of Eq. (22), and formally can be viewed as its particular case. Various asymptotically equivalent versions of Eq. (22) can be considered, using leading-order relations $u_{x x}=\left[u_{t t}+E_{4}(u\right.$ $-w)] / c_{0}^{2}+\ldots, \quad w_{x x}=\left[w_{t t}+\hat{E}_{4}(w-u)\right] / \hat{c}_{0}^{2}+\ldots$. Based on the shown similarity between continuum models for our basic lattice model and a macroscopic waveguide (see Sec. II), we believe that similar description can be obtained within the framework of dynamic nonlinear elasticity. In what follows we focus on the properties of cRB Eq. (21).

\section{B. Conservation laws}

We find conservation laws for the cRB Eqs. (21), which are used to control the accuracy of numerical simulations of their solutions.

We can rescale variables in Eq. (21) as

$$
t \rightarrow \frac{t}{T^{*}}, \quad x \rightarrow \frac{x}{X^{*}}, \quad u \rightarrow \frac{u}{U^{*}}, \quad w \rightarrow \frac{w}{U^{*}},
$$

with

$$
U^{*}=\frac{c_{0}^{2} X^{*}}{E_{1}}, \quad T^{*}=\frac{X^{*}}{c_{0}}, \quad X^{*}=\left(E_{2}+\frac{E_{3}}{c_{0}^{2}}\right)^{1 / 2}
$$

to write Eqs. (21) in the form

$$
\begin{gathered}
u_{t t}-u_{x x}=u_{x} u_{x x}+u_{t t x x}-\delta(u-w), \\
w_{t t}-c^{2} w_{x x}=\alpha w_{x} w_{x x}+\beta w_{t t x x}+\gamma(u-w) .
\end{gathered}
$$

The coefficients in Eqs. (23) are given by

$$
\begin{aligned}
& c^{2}=\frac{\hat{c}_{0}^{2}}{c_{0}^{2}}, \quad \delta=\left(E_{2}+\frac{E_{3}}{c_{0}^{2}}\right) \frac{E_{4}}{c_{0}^{2}}, \quad \alpha=\frac{\hat{E}_{1}}{E_{1}}, \\
& \beta=\left(\hat{E}_{2}+\frac{\hat{E}_{3}}{\hat{c}_{0}^{2}}\right) /\left(E_{2}+\frac{E_{3}}{c_{0}^{2}}\right), \quad \gamma=\frac{\delta \hat{E}_{4}}{E_{4}} .
\end{aligned}
$$

The system (23) is Lagrangian with the density

$$
\begin{aligned}
L= & \frac{1}{2}\left[u_{t}^{2}+\frac{\delta}{\gamma} w_{t}^{2}-u_{x}^{2}-\frac{\delta c^{2}}{\gamma} w_{x}^{2}-\frac{1}{3}\left(u_{x}^{3}+\frac{\alpha \delta}{\gamma} w_{x}^{3}\right)+u_{t x}^{2}\right. \\
& \left.+\frac{\beta \delta}{\gamma} w_{t x}^{2}-\delta(u-w)^{2}\right] .
\end{aligned}
$$

In addition to a simple conservation law

$$
\begin{aligned}
& {\left[u_{t}+\frac{\delta}{\gamma} w_{t}\right]_{t}-\left[u_{x}+\frac{\delta c^{2}}{\gamma} w_{x}+\frac{1}{2} u_{x}^{2}+\frac{\alpha \delta}{2 \gamma} w_{x}^{2}+u_{t t x}+\frac{\beta \delta}{\gamma} w_{t t x}\right]_{x}} \\
& \quad=0
\end{aligned}
$$

immediately following from Eqs. (23), it has two nontrivial conservation laws, for energy and momentum,

$$
A_{t}^{i}+B_{x}^{i}=0, \quad i=1,2,
$$

where the respective densities and flows are given by

$$
\begin{aligned}
A^{1}= & \frac{1}{2}\left[u_{t}^{2}+\frac{\delta}{\gamma} w_{t}^{2}+u_{x}^{2}+\frac{\delta c^{2}}{\gamma} w_{x}^{2}+\frac{1}{3}\left(u_{x}^{3}+\frac{\alpha \delta}{\gamma} w_{x}^{3}\right)+u_{t x}^{2}\right. \\
& \left.+\frac{\beta \delta}{\gamma} w_{t x}^{2}+\delta(u-w)^{2}\right], \\
B^{1}=- & u_{t} u_{x}-\frac{\delta c^{2}}{\gamma} w_{t} w_{x}-\frac{1}{2} u_{t} u_{x}^{2}-\frac{\alpha \delta}{2 \gamma} w_{t} w_{x}^{2}-u_{t} u_{t t x}-\frac{\beta \delta}{\gamma} w_{t} w_{t t x}, \\
B^{2}= & -\frac{1}{2}\left[u_{t}^{2}+\frac{\delta}{\gamma} w_{t}^{2}+u_{x}^{2}+\frac{\delta c^{2}}{\gamma} w_{x}^{2}+\frac{\delta}{3}\left(w_{t} w_{x}+u_{t x} u_{x x}+\frac{\beta \delta}{\gamma} w_{t x} w_{x x},\right.\right. \\
& \left.+\frac{\beta \delta}{\gamma} w_{t x}^{2}-\delta(u-w)^{2}\right]-u_{x} u_{t t x}-\frac{\beta \delta}{\gamma} w_{x} w_{t t x} .
\end{aligned}
$$

Indeed, one can verify that Eqs. (24) hold by virtue of Eqs. (23).

\section{CLASSICAL AND GENERALIZED SOLITARY WAVES}

Differentiating system (23) with respect to $x$ and denoting $u_{x}=f, w_{x}=g$, we obtain

$$
\begin{gathered}
f_{t t}-f_{x x}=\frac{1}{2}\left(f^{2}\right)_{x x}+f_{t t x x}-\delta(f-g), \\
g_{t t}-c^{2} g_{x x}=\frac{1}{2} \alpha\left(g^{2}\right)_{x x}+\beta g_{t t x x}+\gamma(f-g) .
\end{gathered}
$$

In the symmetric case when $c=\alpha=\beta=1$, system (25) admits a reduction $g=f$, where $f$ satisfies the equation

$$
f_{t t}-f_{x x}=\frac{1}{2}\left(f^{2}\right)_{x x}+f_{t t x x},
$$

same as for a single waveguide (see Sec. II B).

Equation (26) has particular solutions in the form of solitary waves, 

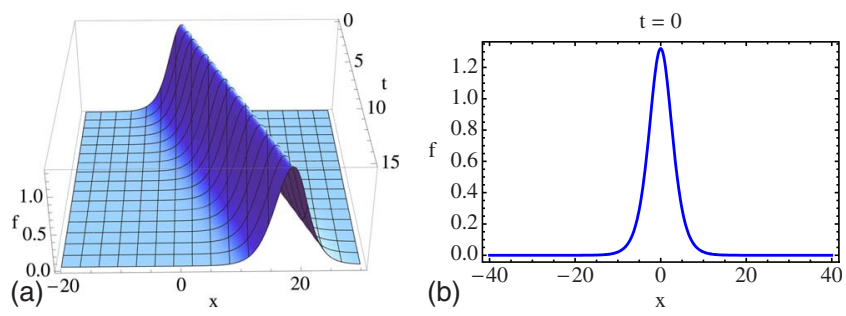

FIG. 5. (Color online) Solitary wave (27) for $v=1.2$.

$$
f=A \operatorname{sech}^{2} \frac{x-v t}{\Lambda}
$$

where $A=3\left(v^{2}-1\right), \quad \Lambda=\frac{2 v}{\sqrt{v^{2}-1}}$ shown in Fig. 5 for $v=1.2$.

It turns out that these pure or classical solitary wave solutions, rapidly decaying to zero in their tail regions (e.g., $[55,56])$, are structurally unstable and are replaced with socalled generalized solitary waves with a copropagating oscillatory tail (see [57-68] and references therein), as shown below.

\section{A. Linear dispersion relation and generalized solitary waves}

We consider system (23) and assume that coefficients are perturbed compared to the symmetric case above but remain positive. The linear dispersion relation is obtained by seeking solutions of the linearized equations in the form

$$
f=A e^{i k(x-p t)}+\text { c.c. }, \quad g=B e^{i k(x-p t)}+\text { c.c. },
$$

where $k$ is a wave number and $p$ is a phase velocity, resulting in

$$
\left[k^{2}\left(1-p^{2}\right)-k^{4} p^{2}+\delta\right]\left[k^{2}\left(c^{2}-p^{2}\right)-\beta k^{4} p^{2}+\gamma\right]=\gamma \delta .
$$

The dispersion curve is symmetric with respect to both $k$ and $p$, and we will consider it only in the first quadrant, where the curve has two branches. Although Eq. (28) is a biquadratic equation, solving it directly is of little help as the resulting formula is hard to analyze. It turns out that the analysis of Eq. (28) is considerably simplified if we make a change of variable

$$
S=k^{2}\left(1-p^{2}\right)-k^{4} p^{2}+\delta, \quad \text { or } p^{2}=\frac{\delta+k^{2}-S}{k^{2}\left(1+k^{2}\right)},
$$

and rewrite the dispersion relation (28) as

$$
S^{2}-b S-c=0 \text {, }
$$

where

$$
\begin{gathered}
b=\delta+k^{2}-\kappa\left(\gamma+k^{2} c^{2}\right), \quad c=\kappa \gamma \delta, \\
\kappa=\left(1+k^{2}\right) /\left(1+\beta k^{2}\right) .
\end{gathered}
$$

We also have the following relation between the amplitudes $A$ and $B$ :

$$
B=\frac{S}{\delta} A=\frac{\kappa \gamma}{S-b} A
$$

From Eq. (30)

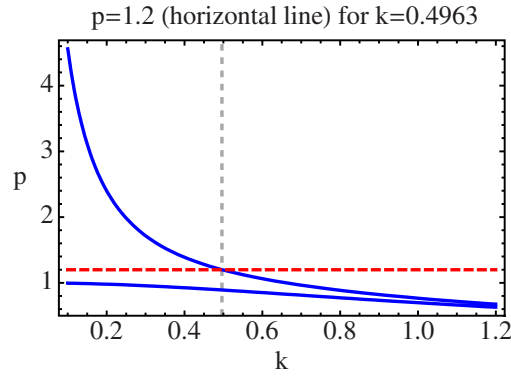

FIG. 6. (Color online) Two branches of the linear dispersion curve of Eq. (23) for $c=1, \beta=1.1, \delta=\gamma=0.1$, and intersection with $p=1.2$ (horizontal line).

$$
S_{1,2}=\frac{1}{2}\left(b \pm \sqrt{b^{2}+4 c}\right),
$$

and we conclude that $S_{1}>0$ and $S_{2}<0$, since $c>0$. Moreover, one can show that $0<S_{1} \leq \delta+k^{2}$; therefore the dispersion relation yields real values of $p$ for all real wave numbers $k$ for both branches of the dispersion relation and the linearized system is spectrally stable. Further, for any fixed value of $k$ we have $S_{2}<S_{1}$. Then from Eq. (29), $p_{2}>p_{1}$, and we conclude that the two branches do not intersect at any finite value of $k$. Finally, analyzing the behavior of the branches as $k \rightarrow 0$ and $k \rightarrow \infty$, we find the following asymptotics:

$$
\begin{gathered}
p_{1} \rightarrow \sqrt{\frac{\gamma+\delta c^{2}}{\gamma+\delta}}+O\left(k^{2}\right), \quad \text { as } k \rightarrow 0, \\
p_{2} \rightarrow \frac{\sqrt{\gamma+\delta}}{k}+O(k), \quad \text { as } k \rightarrow 0,
\end{gathered}
$$

and

$$
\begin{aligned}
& p_{1} \rightarrow \begin{cases}\frac{c}{\sqrt{\beta} k}+O\left(\frac{1}{k^{3}}\right), & \text { if } \beta>c^{2}, \\
\frac{1}{k}+O\left(\frac{1}{k^{3}}\right), & \text { if } \beta<c^{2},\end{cases} \\
& p_{2} \rightarrow \begin{cases}\frac{1}{k}+O\left(\frac{1}{k^{3}}\right), & \text { if } \beta>c^{2}, \\
\frac{c}{\sqrt{\beta} k}+O\left(\frac{1}{k^{3}}\right), & \text { if } \beta<c^{2} .\end{cases}
\end{aligned}
$$

A typical linear dispersion curve of Eq. (23) is shown in Fig. 6. Significant difference with the linear dispersion curve of the reduction (26) in the symmetric case (shown in Fig. 7) consists in the appearance of the second (upper) branch, going to infinity as $k \rightarrow 0$, and approaching zero, remaining above the lower branch, as $k \rightarrow \infty$.

The classical or pure solitary waves of the symmetric case arise as a bifurcation from wave number $k=0$ of the linear wave spectrum shown in Fig. 7, when there is no possible resonance with any linear wave for any value of $k$. The solitary wave speed $v$ is greater than the linear long-wave speed, i.e., $v>1$, while the speed of linear wave of any wave number is smaller, i.e., $p \leq 1$. This becomes impossible when 


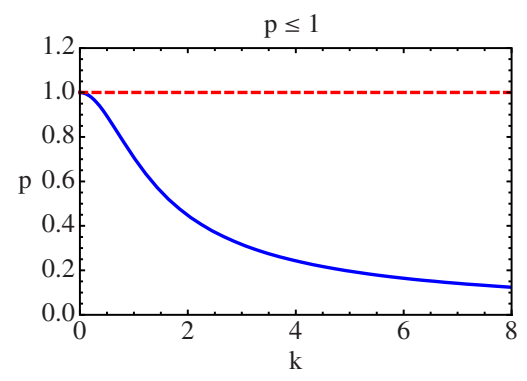

FIG. 7. (Color online) Linear dispersion curve of the reduction (26) in the symmetric case $c=1, \beta=1$, and $\delta=\gamma=0.1$.

the symmetry is broken. Instead, generalized solitary waves arise as a bifurcation from the lower dispersion curve branch at wave number $k=0$ for the case when there is a possible resonance with the upper branch for some finite nonzero value of $k$. For example, a possible resonance is shown in Fig. 6 for $v=p=1.2$. The generalized solitary wave clearly manifests itself in numerical simulations discussed in the next section.

\section{B. Numerical simulations}

In this section, we discuss numerical simulations of generalized solitary wave solutions of the system (25). We break the symmetry of the system (25) with $c=\alpha=\beta=1$ and $\gamma=\delta$ by perturbing the coefficients $\beta$ and $c$, while keeping $\alpha=1$ and $\gamma=\delta$. This is a natural perturbation related to the dispersive properties of the layered structure. We numerically integrate Eq. (25) with the perturbed coefficients, imposing the initial condition (27) on both $f$ and $g$. Our emphasis will be on verifying appearance of ripples moving with the speed of the core solitary wave. We will also compare numerically calculated wavelength and period of the ripples with the theoretical predictions made in the previous section.

We use a Fourier basis for the $x$ dependence of $f$ and $g$. Then, writing any variable $q(x, t)$ as

$$
q(x, t)=\sum_{j \geq 0} \tilde{q}_{j}(t) e^{i j x}+\text { c.c. },
$$

where $j$ is one of the numerically resolved wave numbers, we write Eq. (25) as the first-order system

$$
\left(\begin{array}{c}
\tilde{f} \\
\tilde{f}_{t} \\
\tilde{g} \\
\tilde{g}_{t}
\end{array}\right)_{t}=\left(\begin{array}{cccc}
0 & 1 & 0 & 0 \\
-\frac{j^{2}}{1+j^{2}} & 0 & 0 & 0 \\
0 & 0 & 0 & 1 \\
0 & 0 & -\frac{j^{2} c^{2}}{1+\beta j^{2}} & 0
\end{array}\right)\left(\begin{array}{c}
\tilde{f} \\
\tilde{f}_{t} \\
\tilde{g} \\
\tilde{g}_{t}
\end{array}\right)
$$

where the terms $\tilde{F}_{j}(t)$ and $\widetilde{G}_{j}(t)$ originate from

$$
\begin{gathered}
F(x, t)=\frac{1}{2}\left(f^{2}\right)_{x x}-\delta(f-g), \\
G(x, t)=\frac{1}{2} \alpha\left(g^{2}\right)_{x x}+\gamma(f-g) .
\end{gathered}
$$

We solve Eq. (33) using a split-step scheme (see, for example, $[69,70])$. Neglecting the terms involving $\widetilde{F}_{j}$ and $\widetilde{G}_{j}$ in Eq. (33), we obtain a linear system that can be updated exactly, since given $\tilde{f}, \tilde{f}_{t}, \widetilde{g}$, and $\widetilde{g}_{t}$ at time $t$, we can write down explicitly the solution of the linear system at time $t+\Delta t$. Similarly, neglecting the terms not involving $\widetilde{F}_{j}$ and $\widetilde{G}_{j}$ in Eq. (33), we obtain a system in which $\tilde{f}$ and $\widetilde{g}$ are constant, so that $\tilde{f}_{t}$ and $\widetilde{g}_{t}$ can be trivially updated by using the values of
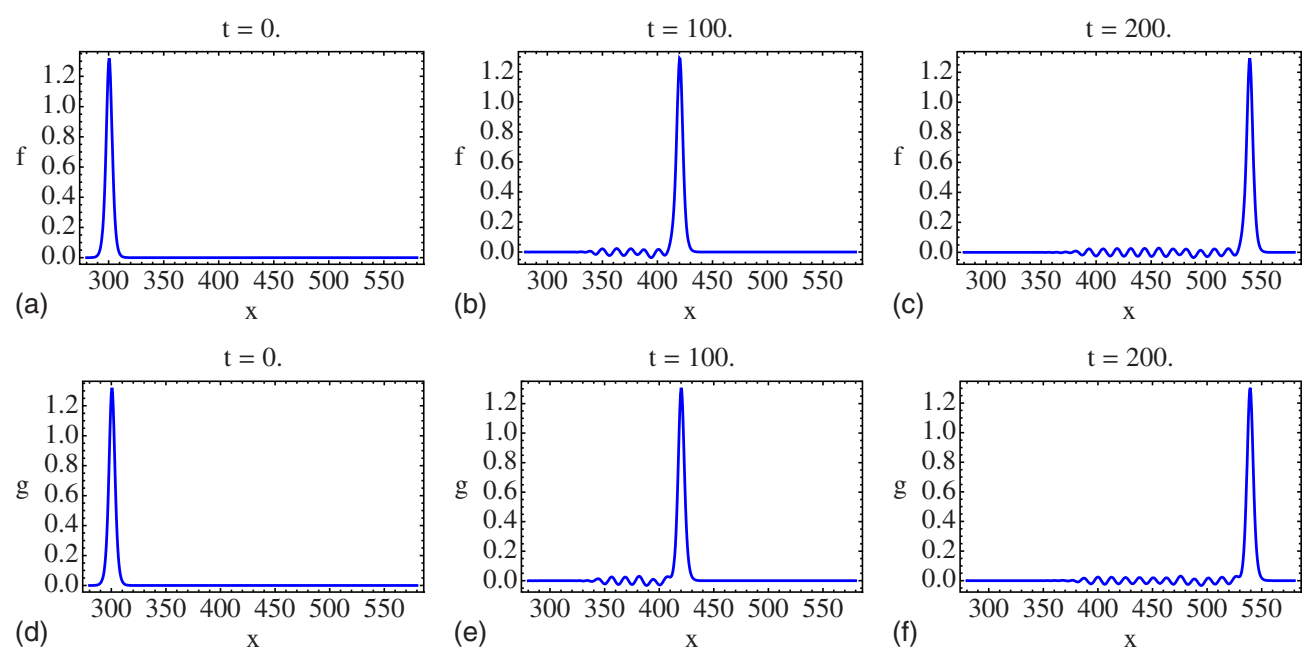

FIG. 8. (Color online) Generation of a generalized solitary wave for $c=1, \alpha=1, \beta=1.1$, and $\gamma=\delta=0.1$; $v=1.2$, from a classical solitary wave of the symmetric case $\left(n_{x}=1024, n_{t}=500\right)$. 
f

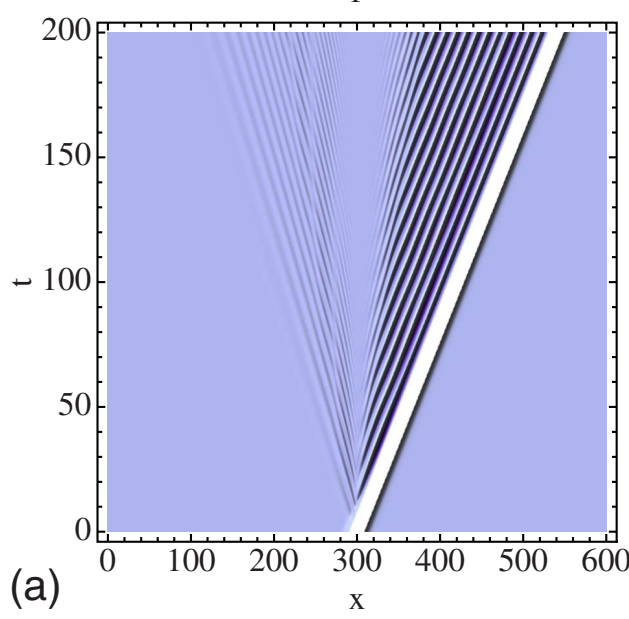

$\mathrm{g}$

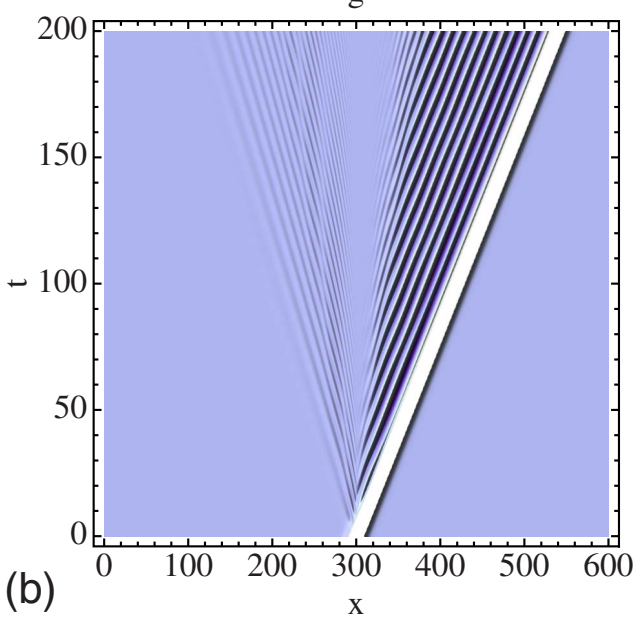

FIG. 9. (Color online) Generation of a generalized solitary wave for $c=1, \alpha=1, \beta=1.1$, and $\gamma=\delta=0.1 ; v=1.2\left(n_{x}=1024, n_{t}=500\right)$. Plot range: $f \in[\min f \approx-0.039$ (black), 0.039 (white) $], g \in[\min g$ $\approx-0.039$ (black), 0.039 (white) $]$. The core solitary wave is clipped at $f=0.039, \quad g=0.039$.

$\widetilde{F}_{j}$ and $\widetilde{G}_{j}$ at time $t$. By combining these two steps in a sequence of appropriately chosen fractional steps, one may obtain a method of any order (e.g., [69]). For the simulations to be presented, we use a fourth-order scheme with 500 to 2000 time steps $n_{t}$, and with 1024 to 4096 points $n_{x}$ in the $x$ direction, and the terms $\widetilde{F}_{j}$ and $\widetilde{G}_{j}$ evaluated pseudospectrally. Conservation of energy and momentum (24) has been monitored, with maximum deviations from the mean value being in the range of $0.4 \%$ and mean squared deviations at about $0.02 \%$. The amplitude of the ripples was typically at least $2 \%$ from the amplitude of the core solitary wave.

The generation of a generalized solitary wave in both components $f$ and $g$ is shown in Fig. 8 for $c=1, \alpha=1, \beta$ $=1.1$, and $\gamma=\delta=0.1 ; v=1.2$. The initial conditions in the form of two identical solitons in both $f$ and $g$ are shown in the left panel. As the waves propagate to the right, the ripples are formed behind the core solitary waves shown in the central and the right panels for $t=100$ and $t=200$, respectively.

Note that importantly, after a brief transient period, the accompanying ripples start moving at the velocity of the core
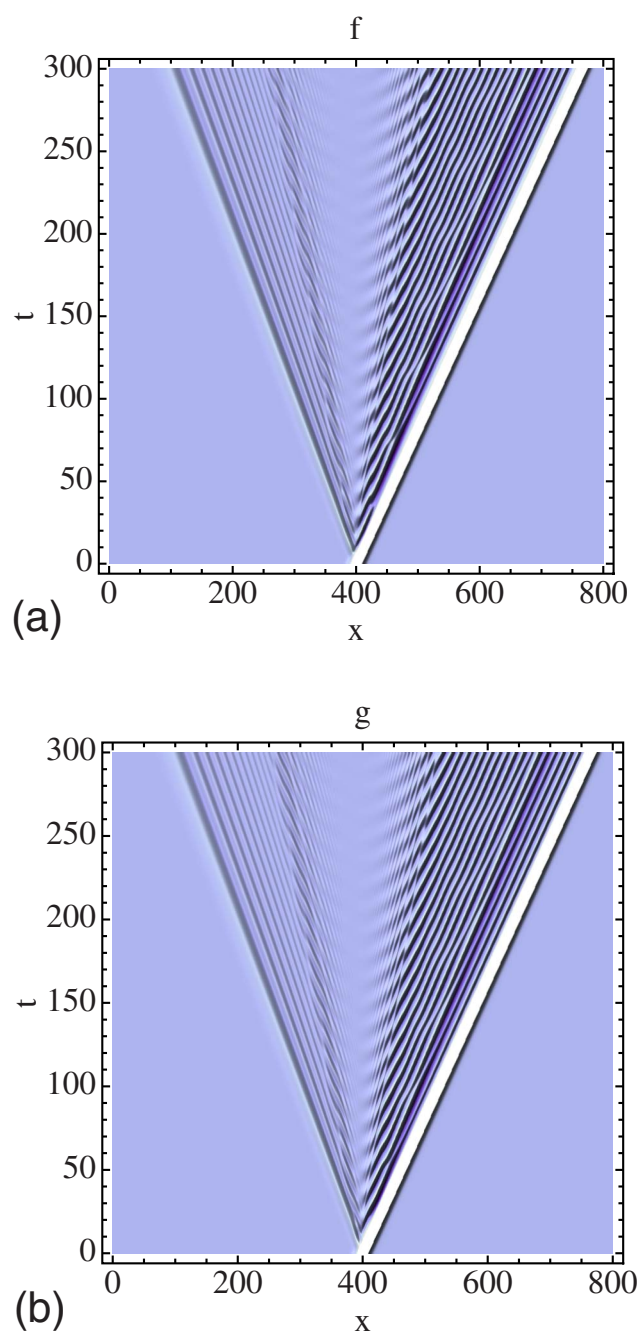

FIG. 10. (Color online) Generation of a generalized solitary wave for $c=1.05, \alpha=1, \beta=1.05$, and $\gamma=\delta=0.1 ; v=1.2\left(n_{x}=1024\right.$, $n_{t}=500$ ). Plot range: $f \in[\min f \approx-0.032$ (black), 0.032 (white) $], g$ $\in[\min g \approx-0.036$ (black), 0.036 (white) $]$. The core solitary wave is clipped at $f=0.032, g=0.036$.

solitary wave, which is best seen in the relief plots shown in Fig. 9 for the same values of physical parameters as in Fig. 8 . In these plots, the emphasis is on the small amplitude structures formed behind the core solitary wave, which is clipped at $f=g=0.039$ (shown by white color). The periodic wave behind the core solitary wave is seen as an alternating sequence of light and dark lines, which after a brief transient process become parallel to the trajectory of the core solitary wave. One can also notice that the periodic waves in the tails of $f$ and $g$ have opposite sign, which is in agreement with formula (31).

Figure 10 shows the formation of a generalized solitary wave for $c=1.05, \alpha=1, \beta=1.05$, and $\gamma=\delta=0.1 ; v=1.2$. Note that the perturbation in $c$ significantly increases the duration of the transient period, which is now accompanied with quite noticeable radiation to the left. Nonetheless, after this transient period one can again notice the formation of a generalized solitary wave similar to that shown in Fig. 9.

In Table I we compare numerically calculated values of the period $T_{n}$ and the wavelength $\lambda_{n}$ of the ripples with the 
TABLE I. Numerically determined period $T_{n}$ and wavelength $\lambda_{n}$ of a copropagating wave (only approximately constant for each numerically found solution) for $c=1, \alpha=1, \beta=1.1$, and $\gamma=\delta$ versus rounded theoretical predictions $T$ and $\lambda\left(n_{x}=4096, n_{t}=2000\right)$.

\begin{tabular}{cccc}
\hline \hline & \multicolumn{3}{c}{$v$} \\
\cline { 2 - 4 }$\delta$ & 1.1 & 1.2 & 1.3 \\
\hline \multirow{2}{*}{0.05} & $T_{n}=12.5 \pm 0.1$ & $T_{n}=13.5 \pm 0.1$ & $T_{n}=14.4 \pm 0.1$ \\
& $T \approx 12.47$ & $T \approx 13.52$ & $T \approx 14.41$ \\
& $\lambda_{n}=13.60 \pm 0.15$ & $\lambda_{n}=16.55 \pm 0.15$ & $\lambda_{n}=18.31 \pm 0.15$ \\
& $\lambda \approx 13.71$ & $\lambda \approx 16.22$ & $\lambda \approx 18.74$ \\
0.1 & $T_{n}=10.1 \pm 0.1$ & $T_{n}=10.5 \pm 0.1$ & $T_{n}=11.0 \pm 0.1$ \\
& $T \approx 10.05$ & $T \approx 10.55$ & $T \approx 10.99$ \\
& $\lambda_{n}=10.99 \pm 0.15$ & $\lambda_{n}=12.60 \pm 0.15$ & $\lambda_{n}=14.5 \pm 0.15$ \\
& $\lambda \approx 11.06$ & $\lambda \approx 12.66$ & $\lambda \approx 14.28$ \\
0.15 & $T_{n}=8.9 \pm 0.1$ & $T_{n}=9.2 \pm 0.1$ & $T_{n}=9.4 \pm 0.1$ \\
& $T \approx 8.91$ & $T \approx 9.21$ & $T \approx 9.47$ \\
& $\lambda_{n}=9.80 \pm 0.15$ & $\lambda_{n}=10.99 \pm 0.15$ & $\lambda_{n}=12.30 \pm 0.15$ \\
& $\lambda \approx 9.81$ & $\lambda \approx 11.05$ & $\lambda \approx 12.31$ \\
\hline \hline
\end{tabular}

theoretical predictions $T=2 \pi /\left(k p_{2}\right)$ and $\lambda=2 \pi / k$ of the previous subsection, for $c=1, \alpha=1, \beta=1.1$, and several values of parameters $\gamma=\delta$ and $v$. We note that a copropagating wave is only approximately periodic; therefore numerical values are shown as values in a certain interval. Generally, agreement between numerical values and theoretical predictions is quite good, especially for the period of waves. Also, a copropagating wave becomes shorter with the increase of $\delta$ or decrease of $v$, in agreement with the theory. Thus, numerical simulations of this section confirm that stable generalized solitary wave solutions of the cRB Eqs. (25) appear in the long-time asymptotics of localized initial data, replacing classical solitary waves of a single regularized Boussinesq Eq. (26).

\section{CONCLUDING REMARKS}

We studied nonlinear longitudinal waves in a two-layered structure with a soft bonding layer using a relatively simple long-wave model in the form of coupled Boussinesq-type equations. The derivation of this model was based on a nonlinear layered dipole lattice model, having all essential degrees of freedom of a realistic layered structure. Moreover, this approach allowed us to consider both geometrical and physical sources of nonlinearity in the structure. Although the derivations were rather challenging and required symbolic computations, they seem to be easier and physically more transparent than similar derivations within the scope of dynamic elasticity theory for nonlinearly elastic materials (e.g., the Murnaghan model [71,72]), which are yet to be performed. Nevertheless, based on the shown similarity between long-wave models for a single lattice waveguide and a nonlinearly hyperelastic waveguide, we conjectured that a similar wave regime described in terms of coupled Boussinesq-type equations exists within the scope of nonlin- ear dynamic elasticity. Indeed, we found explicit formulas relating parameters of the nonlinear dipole lattice with the elastic moduli of the continuum.

Our study reveals an important difference in the properties of nonlinear waves in a layered structure with an imperfect interface compared to the limiting case of the perfect (or ideal) interface (which can be considered as a single homogeneous waveguide). We showed that the classical solitary wave solution, which exists in a homogeneous waveguide is generally replaced with a generalized solitary wave accompanied by a copropagating periodic wave in its tail. This effect may be related to the recently observed increase in the decay rate of a nonlinear longitudinal bulk strain solitary wave in "imperfectly" bonded layered structure compared to the decay rate in a comparable homogeneous waveguide or "perfectly" bonded layered structure [27]. Indeed, in the generalized solitary wave, the amplitude of the core solitary wave gradually decreases due to the continuous radiation of a copropagating wave. As the model considered in this paper is rather robust, we would expect similar phenomena to take place in other physical contexts, where the model could be applied.

\section{ACKNOWLEDGMENTS}

We thank E. S. Benilov, R. H. J. Grimshaw and A. B. Movchan for useful discussions. The research was supported by the UK EPSRC under Grant No. EP/D035570/1.

\section{APPENDIX A}

The nonzero coefficients are given in terms of the lattice parameters by the following formulas:

$$
\begin{gathered}
b_{14}=-b_{23}=\beta_{1} \sin 2 \alpha, \quad b_{i j}=-b_{j i}, \\
c_{11}=2\left(\beta+\beta_{1} \cos ^{2} \alpha\right), \quad c_{22}=-c_{44}=2 \beta_{1} \sin ^{2} \alpha, \\
c_{33}=2\left(\beta-\beta_{1} \cos ^{2} \alpha\right) ; \quad d_{33}=-8 \beta_{1} \cos ^{2} \alpha, \\
d_{44}=-8 \beta_{1} \sin ^{2} \alpha-4 \beta_{\perp}, \\
g_{111}=2\left(\gamma+\gamma_{1} \cos ^{3} \alpha\right)+\frac{3 \beta_{1}}{d} \cos \alpha \sin ^{2} \alpha, \\
g_{122}=g_{212}=g_{144} \\
=2 \gamma_{1} \cos \alpha \sin ^{2} \alpha+\frac{\beta}{d \cos \alpha}+\frac{\beta_{1}}{d} \cos ^{3} \alpha \\
-\frac{2 \beta_{1}}{d} \cos \alpha \sin ^{2} \alpha, \\
g_{133}=g_{111}-\frac{2 \beta_{1}}{d} \cos \alpha, \quad g_{234}=-g_{122}-\frac{2 \beta_{1}}{d} \cos \alpha, \\
g_{313}=-g_{111}+4 \gamma,
\end{gathered}
$$




$$
\begin{aligned}
& g_{324}=-g_{414}=g_{423}=g_{122}-\frac{2 \beta}{d \cos \alpha}, \quad g_{i j k}=g_{i k j}, \\
& h_{123}=-h_{114}=h_{213}=-h_{312}=h_{411}=-\gamma_{1} \sin \alpha \cos ^{2} \alpha \\
& +\frac{\beta_{1}}{d} \sin \alpha \cos ^{2} \alpha-\frac{\beta_{1}}{2 d} \sin ^{3} \alpha, \\
& h_{224}=-h_{422}=-h_{444}=\gamma_{1} \sin ^{3} \alpha+\frac{3 \beta_{1}}{2 d} \sin \alpha \cos ^{2} \alpha, \\
& h_{334}=h_{123}+\frac{\beta}{d \sin \alpha}-\frac{\beta_{1}}{d \sin \alpha} \cos ^{2} \alpha, \\
& h_{433}=h_{411}+\frac{\beta_{1}}{d} \sin \alpha, \quad h_{i j k}=h_{i k j}, \\
& l_{12}=-8 \gamma_{1} \sin \alpha \cos ^{2} \alpha+\frac{8 \beta_{1}}{d} \sin \alpha \cos ^{2} \alpha-\frac{4 \beta_{1}}{d} \sin ^{3} \alpha, \\
& l_{34}=l_{12}+\frac{4 \beta}{d \sin \alpha}-\frac{4 \beta_{1}}{d \sin \alpha} \cos 2 \alpha, \quad l_{i j}=l_{j i}, \\
& \tilde{l}_{11}=-l_{12}, \quad \tilde{l}_{22}=-\tilde{l}_{44}=8 \gamma_{1} \sin ^{3} \alpha+\frac{12 \beta_{1}}{d} \sin \alpha \cos ^{2} \alpha, \\
& \tilde{l}_{33}=l_{12}+\frac{8 \beta}{d \sin \alpha}-\frac{8 \beta_{1}}{d \sin \alpha} \cos ^{2} \alpha, \\
& m_{13}=8 \gamma_{1} \cos ^{3} \alpha-\frac{4 \beta_{1}}{d} \cos \alpha+\frac{12 \beta_{1}}{d} \sin ^{2} \alpha \cos \alpha, \\
& m_{24}=-8 \gamma_{1} \sin ^{2} \alpha \cos \alpha-\frac{4 \beta_{1}}{d} \cos \alpha-\frac{4 \beta_{1}}{d} \cos ^{3} \alpha \\
& +\frac{8 \beta_{1}}{d} \sin ^{2} \alpha \cos \alpha, \quad m_{i j}=-m_{j i}, \\
& \tilde{m}_{14}=-m_{24}-\frac{4 \beta_{1}}{d} \cos \alpha, \quad \tilde{m}_{23}=m_{24}, \quad \tilde{m}_{i j}=-\tilde{m}_{j i}, \\
& p_{334}=p_{433}=2 l_{12}-\frac{16 \beta_{1}}{d \sin \alpha} \cos ^{2} \alpha+\frac{8 \beta_{1}}{d} \sin \alpha, \\
& p_{444}=-16 \gamma_{1} \sin ^{3} \alpha-\frac{24 \beta_{1}}{d} \sin \alpha \cos ^{2} \alpha-8 \gamma_{\perp}, \\
& p_{i j k}=p_{i k j}
\end{aligned}
$$

\section{APPENDIX B}

The truncated nonlinear terms $N_{1-4}$ in the right-hand side of Eqs. (7) are given by

$$
\begin{aligned}
N_{i}= & g_{i j k}\left(a^{2} u_{j_{x x}}+\frac{a^{4}}{12} u_{j_{x x x x}}\right)\left(2 a u_{k_{x}}+\frac{a^{3}}{3} u_{k_{x x x}}\right) \\
& +\left(l_{i j} u_{3}+\tilde{l}_{i j} u_{4}\right)\left(a^{2} u_{j_{x x}}+\frac{a^{4}}{12} u_{j_{x x x x}}\right) \\
& +h_{i j k}\left[\left(a^{2} u_{j_{x x}}+\frac{a^{4}}{12} u_{j_{x x x x}}\right)\left(a^{2} u_{k_{x x}}+\frac{a^{4}}{12} u_{k_{x x x x}}\right)\right. \\
& \left.+\left(2 a u_{j_{x}}+\frac{a^{3}}{3} u_{j_{x x x}}\right)\left(2 a u_{k_{x}}+\frac{a^{3}}{3} u_{k_{x x x}}\right)\right]+\left(m_{i j} u_{3}+\tilde{m}_{i j} u_{4}\right) \\
& \times\left(2 a u_{j_{x}}+\frac{a^{3}}{3} u_{j_{x x x}}\right)+p_{i j k} u_{j} u_{k} .
\end{aligned}
$$

\section{APPENDIX C}

Weak (i.e., the higher order in $\varepsilon$ ) transversal and rotational motions are slaved to longitudinal waves, being governed by the subsystem of equations for $\widetilde{u}_{2}$ and $\widetilde{u}_{3}$,

$$
\begin{aligned}
& M v^{2} \widetilde{u}_{2_{\pi t}}+a^{2}\left(2 b_{14} \widetilde{u}_{\tilde{x}_{\tilde{x}}}-c_{22} \widetilde{u}_{2_{\widetilde{x}}}\right)-\varepsilon a^{3}\left[2 m_{24}\left(\widetilde{u}_{3} \widetilde{u}_{4_{\tilde{x}}}+\widetilde{u}_{3_{\tilde{x}}} \tilde{u}_{4}\right)\right.
\end{aligned}
$$

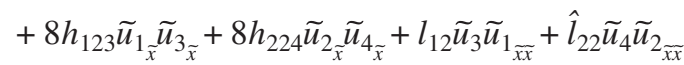

$$
\begin{aligned}
& \left.+2 g_{122}\left(\widetilde{u}_{1_{\tilde{x}}} \widetilde{u}_{2 \widetilde{x x}}+\widetilde{u}_{1_{\widehat{x x}}} \widetilde{u}_{\tilde{x}_{\tilde{x}}}\right)\right]+\varepsilon a^{2}\left(\frac{b_{14}}{3} \widetilde{u}_{3 \widetilde{x x x}}-\frac{c_{22}}{12} \widetilde{u}_{2_{\overparen{x x x x}}}\right) \\
& +\mathcal{O}\left(\varepsilon^{2}\right)=0 \\
& 2 b_{14} \widetilde{u}_{2_{\tilde{x}}}+d_{33} \widetilde{u}_{3}+\varepsilon\left[-\frac{M v^{2}}{a^{2}} \widetilde{u}_{3 \widetilde{t t}}+c_{33} \widetilde{u}_{3 \widetilde{x x}}+\frac{b_{14}}{3} \widetilde{u}_{2 \widetilde{x x x}}\right. \\
& \left.+2 a\left(p_{334} \widetilde{u}_{3} \widetilde{u}_{4}-m_{13} \widetilde{u}_{3} \widetilde{u}_{1 \tilde{x}}-m_{24} \widetilde{u}_{4} \widetilde{u}_{2_{\tilde{x}}}-4 h_{123} \widetilde{u}_{1_{\tilde{x}}} \widetilde{u}_{\tilde{x}_{\tilde{x}}}\right)\right] \\
& +\mathcal{O}\left(\varepsilon^{2}\right)=0 .
\end{aligned}
$$

Eliminating $\widetilde{u}_{3}$, we derive an equation for $\widetilde{u}_{2}$, which in dimensional variables has the form

$$
u_{2 t t}-c_{0}^{2}\left(u_{2_{x}} u_{1_{x x}}+u_{2_{x x}} u_{1_{x}}\right)-l^{2} u_{2_{t t x x}}+\frac{2 a^{2} l^{2}}{M} \beta u_{2_{x x x x}}=0 .
$$

To leading order, the rotational motions are defined by $u_{3}$ $=l u_{2_{x}}+\ldots$.

\section{APPENDIX D}

The subsystem for longitudinal waves follows from the equations for $\widetilde{u}_{1}, \widetilde{u}_{4}$ and $\widetilde{u}_{5}$ and has the form:

$$
\begin{aligned}
& M v^{2} \widetilde{u}_{5_{\tilde{t}}}=3 a^{2}\left[\left(\beta+\beta_{1} \cos ^{2} \alpha\right) \widetilde{u}_{\widetilde{x x}_{\overparen{x}}}-2 \beta_{1} \cos ^{2} \alpha \widetilde{w}_{1}\right] \\
& -\varepsilon\left[M v^{2} w_{1 \tilde{t}}+a^{3}\left(D_{3} \tilde{w}_{1} \tilde{u}_{4}+D_{4} \tilde{w}_{1} \tilde{u}_{\tilde{x}}+D_{5} \tilde{u}_{4} \tilde{u}_{\tilde{x x}}\right.\right. \\
& \left.\left.+D_{6} \widetilde{u}_{5_{\tilde{x}}} \widetilde{u}_{5_{x x}}\right)\right]+\varepsilon a^{2}\left(D_{7} \widetilde{w}_{\widehat{x}_{x x}}+D_{8} \widetilde{u}_{\widetilde{u}_{x x x x}}\right)+\mathcal{O}\left(\varepsilon^{2}\right),
\end{aligned}
$$




$$
\begin{aligned}
& 0=\left(3 \beta_{\perp}+6 \beta_{1} \sin ^{2} \alpha\right) \tilde{u}_{4}+3 \beta_{1} \sin 2 \alpha \tilde{u}_{5_{\tilde{x}}} \\
& +\varepsilon\left(\frac{M v^{2}}{a^{2}} \widetilde{u}_{4_{\tilde{t}}}+D_{9} \tilde{u}_{5_{\tilde{x x x}}}+a\left[D_{10} \widetilde{u}_{4}^{2}+D_{11} \widetilde{u}_{4} \widetilde{u}_{5_{\tilde{x}}}\right.\right. \\
& \left.+D_{12}\left(\widetilde{u}_{\tilde{x}^{-}}{ }^{2}\right]\right)+\mathcal{O}\left(\varepsilon^{2}\right), \\
& M v^{2} \widetilde{u}_{5 \pi t}=3 a^{2}\left[4 \beta_{1} \cos ^{2} \alpha \widetilde{w}_{1}+2 \beta_{1} \sin 2 \alpha \tilde{u}_{4_{\tilde{x}}}\right. \\
& \left.+\left(\beta+2 \beta_{1} \cos ^{2} \alpha\right) \widetilde{u}_{5_{\overparen{x x}}}\right]-\varepsilon a^{3}\left(D_{13} \widetilde{w}_{1} \widetilde{u}_{4}+D_{14} \widetilde{w}_{1} \widetilde{u}_{\tilde{x}_{\tilde{x}}}\right.
\end{aligned}
$$

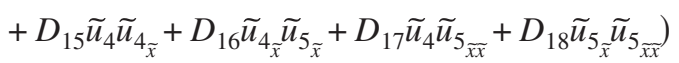

$$
\begin{aligned}
& +\varepsilon a^{2}\left(D_{19} \widetilde{w}_{1_{\widetilde{x x}}}+D_{20} \widetilde{u}_{4 \widetilde{x x x}}+D_{21} \widetilde{u}_{5_{\widetilde{x} x x}}\right)+\mathcal{O}\left(\varepsilon^{2}\right),
\end{aligned}
$$

where $D_{1}-D_{21}$ are some intermediate coefficients. From Eq. (34), using asymptotic reiteration, we find $\widetilde{w}_{1}$ in terms of $\widetilde{u}_{4}$ and $\widetilde{u}_{5}$. Then, $\widetilde{u}_{4}$ can be found from Eq. (35) in terms of $\widetilde{u}_{5}$, again by asymptotic reiteration. After that an equation for $\widetilde{u}_{5}$ is derived from Eq. (36). Returning to dimensional variables, we obtain

$$
u_{5_{t t}}-c_{0}^{2} u_{5_{x x}}=F_{1} u_{5_{x}} u_{5_{x x}}+F_{2} u_{5_{t t x x}}+F_{3} u_{5_{x x x x}}+F_{4} u_{5 t t t t},
$$

where

$$
\begin{aligned}
c_{0}^{2} & =\frac{a^{2}}{M}\left(3 \beta+2 \beta_{\perp} \nu\right), \quad \nu=\frac{2 \beta_{1} \cos ^{2} \alpha}{\beta_{\perp}+2 \beta_{1} \sin ^{2} \alpha}, \\
F_{1}= & \frac{a^{2}}{M}\left\{6 \beta_{\perp} \nu(1+\nu)^{2} \sin ^{2} \alpha\right. \\
& \left.+a\left[6 \gamma-4 \gamma_{\perp} \nu^{3} \tan ^{3} \alpha+\gamma_{1}\left(\frac{\beta_{\perp} \nu}{\beta_{1} \cos \alpha}\right)^{3}\right]\right\},
\end{aligned}
$$

$$
\begin{gathered}
F_{2}=\frac{2}{3}\left[l^{2} \nu^{2}-a^{2}\left(1+\frac{\beta}{\beta_{1} \cos ^{2} \alpha}\right)\right], \\
F_{3}=\frac{a^{4}}{M}\left(\frac{9}{4} \beta+\frac{2}{3} \beta_{\perp} \nu+\frac{\beta^{2}}{\beta_{1} \cos ^{2} \alpha}\right), \quad F_{4}=\frac{M}{9 \beta_{1} \cos ^{2} \alpha},
\end{gathered}
$$

and $u_{4}=-l \nu u_{5}^{\prime}+\ldots$.

Up to $O(\varepsilon)$ terms,

$$
u_{5}=u_{1}+\frac{M}{6 \beta_{1} \cos ^{2} \alpha} u_{1_{t t}}-\frac{a^{2}}{2}\left(1+\frac{\beta}{\beta_{1} \cos ^{2} \alpha}\right) u_{1_{x x}}+\ldots,
$$

which allows one to obtain Eq. (18) from Eq. (37) with the same accuracy, substituting Eq. (38) into the left-hand side of Eq. (37) (i.e., into the leading-order terms).

\section{APPENDIX E}

The relation between $u_{2}$ and $u_{6}$, up to $O(\varepsilon)$ terms, is given by

$$
u_{6}=u_{2}+\frac{M}{3\left(\beta_{\perp}+2 \beta_{1} \sin ^{2} \alpha\right)} u_{t_{t t}}-a^{2} \frac{\beta_{1} \sin ^{2} \alpha}{\beta_{\perp}+2 \beta_{1} \sin ^{2} \alpha} u_{2 x}
$$$$
+\ldots
$$

With the same accuracy, an equation for $u_{6}$ has the form

$$
\begin{aligned}
u_{6_{t t}=} & \frac{2}{3} l^{2}(1-\nu) u_{6_{t t x x}}-\frac{2 a^{2} l^{2}}{M}\left(\beta+\frac{1}{4} \beta_{\perp} \nu\right) u_{6_{x x x x}} \\
& +\frac{\nu M}{9 \beta_{1} \cos ^{2} \alpha} u_{6_{t t t t}}
\end{aligned}
$$

[1] L. Brillouin, Wave Propagation in Periodic Structures (Dover, London, 1953).

[2] A. Askar, Lattice Dynamical Foundations of Continuum Theories (World Scientific, Singapore, 1985).

[3] G. A. Maugin, Nonlinear Waves in Elastic Crystals (Oxford University Press, Oxford, 1999).

[4] O. M. Braun and Y. S. Kivshar, The Frenkel-Kontorova Model: Concepts, Methods, and Applications (Springer-Verlag, Berlin, 2004).

[5] T. A. Kontorova and Ya. I. Frenkel, Zh. Eksp. Teor. Fiz. 8, 89 and 1340 (1938).

[6] S. Takeno, S. V. Dmitriev, P. G. Kevrekidis, and A. R. Bishop, Phys. Rev. B 71, 014304 (2005).

[7] E. Fermi, J. Pasta, and S. Ulam, Los Alamos Scientific Laboratory Report No. LA-1940, 1955; Lect. Appl. Math. 15, 143 (1974).

[8] N. J. Zabusky and M. D. Kruskal, Phys. Rev. Lett. 15, 240 (1965).
[9] M. Toda, J. Phys. Soc. Jpn. 22, 431 (1967).

[10] D. Yagil and T. Kawahara, Wave Motion 34, 97 (2001).

[11] D. H. Yong and R. LeVeque, SIAM J. Appl. Math. 63, 1539 (2003).

[12] A. V. Savin, L. I. Manevich, P. L. Christiansen, and A. V. Zolotaryuk, Phys. Usp. 42, 245 (1999).

[13] K. R. Khusnutdinova, Eur. Phys. J. Spec. Top. 147, 45 (2007).

[14] W. T. Ashurst and W. G. Hoover, Phys. Rev. B 14, 1465 (1976).

[15] E. Smith, Mater. Sci. Eng. 30, 15 (1977).

[16] H. J. Herrmann, A. Hansen, and S. Roux, Phys. Rev. B 39, 637 (1989).

[17] V. V. Ginzburg and L. I. Manevitch, Int. J. Fract. 64, 93 (1993).

[18] J. Astrom and J. Timonen, Phys. Rev. B 54, R9585 (1996).

[19] L. I. Slepyan, Models and Phenomena in Fracture Mechanics (Springer-Verlag, Berlin, 2002).

[20] D. A. Kessler and H. Levine, Phys. Rev. E 68, 036118 (2003). 
[21] T. Martin, P. Español, and M. A. Rubio, Phys. Rev. E 71, 036202 (2005).

[22] L. I. Slepyan and M. V. Ayzenberg-Stepanenko, Int. J. Fract. 140, 235 (2006).

[23] G. S. Mishuris, A. B. Movchan, and L. I. Slepyan, J. Mech. Phys. Solids 56, 487 (2008).

[24] L. B. Freund, Dynamic Fracture Mechanics (Cambridge University Press, Cambridge, England, 1998).

[25] K. R. Khusnutdinova and V. V. Silberschmidt, Proc. Est. Acad. Sci., Phys., Math. 51, 63 (2003).

[26] K. R. Khusnutdinova and A. M. Samsonov, Phys. Rev. E 77, 066603 (2008).

[27] G. V. Dreiden, K. R. Khusnutdinova, A. M. Samsonov, and I. V. Semenova, J. Appl. Phys. 104, 086106 (2008).

[28] G. V. Dreiden, K. R. Khusnutdinova, A. M. Samsonov, and I. V. Semenova, Strain (to be published).

[29] A. C. Eringen, Microcontinuum Field Theories I: Foundations and Solids (Springer, New York, 1999).

[30] R. Phillips, Crystals, Defects, and Microstructures: Modelling Across Scales (Cambridge University Press, Cambridge, England, 2001).

[31] S. Suresh and A. Mortensen, Fundamentals of Functionally Graded Materials (IOM Comm. Ltd, London, 1998).

[32] G. A. Maugin, Material Inhomogeneities in Elasticity (Chapman \& Hall, London, 1993).

[33] K. R. Khusnutdinova, Deep Refinement of Hydrocarbon Material, (TsNIITEneftekhim, Moscow, 1993), Vol. 2, pp. 136-145.

[34] E. A. Il'yushina, Ph.D. thesis, Lomonosov Moscow State University, 1976.

[35] K. R. Khusnutdinova, Vestn. Mosk. Univ., Ser. 1: Mat., Mekh. 2, 71 (1992).

[36] T. D. Dragunov, I. S. Pavlov, and A. I. Potapov, Phys. Solid State 39, 118 (1997).

[37] F. Cote, V. S. Deshpande, N. A. Fleck, and A. G. Evans, Int. J. Solids Struct. 43, 6220 (2006).

[38] Y. Mo, K. T. Turner, and I. Szlufarska, Nature (London) 457, 1116 (2009).

[39] F. Kh. Abdullaev, Yu. V. Bludov, S. V. Dmitriev, P. G. Kevrekidis, and V. V. Konotop, Phys. Rev. E 77, 016604 (2008).

[40] K. R. Khusnutdinova, A. M. Samsonov, and A. S. Zakharov, Theor. Math. Phys. 159, 475 (2009).

[41] MATHEMATICA and WOLFRAM MATHEMATICA are trademarks of Wolfram Research Inc. (www.wolfram.com).

[42] A. M. Samsonov, Sov. Phys. Dokl. 29, 586 (1984).

[43] A. V. Porubov and A. M. Samsonov, Tech. Phys. Lett. 19, 365 (1993).

[44] A. M. Samsonov, Strain Solitons in Solids and How to Construct Them (Chapman and Hall, Boca Raton, 2001).

[45] A. V. Porubov, Amplification of Nonlinear Strain Waves in Solids (World Scientific, Singapore, 2003).
[46] J. Janno and J. Engelbrecht, J. Phys. A 38, 5159 (2005).

[47] V. E. Zakharov, Sov. Phys. JETP 38, 108 (1974).

[48] V. E. Zakharov and A. B. Shabat, Funct. Anal. Appl. 8, 226 (1975).

[49] T. B. Benjamin, J. L. Bona, and J. J. Mahony, Philos. Trans. R. Soc. London, Ser. A 272, 47 (1972).

[50] C. I. Christov, G. A. Maugin, and M. G. Velarde, Phys. Rev. E 54, 3621 (1996).

[51] A. E. H. Love, A Treatise on the Mathematical Theory of Elasticity (Dover, New York, 1944).

[52] E. Volterra and E. C. Zachmanoglou, Dynamics of vibrations (Charles E. Merrill Books, Columbus, 1965).

[53] P. A. Martin, Elastic Waves and Ultrasonic Nondestructive Evaluation (North-Holland, Amsterdam, 1990), pp. 217-222.

[54] G. S. Mishuris, N. V. Movchan, and A. B. Morchan, Q. J. Mech. Appl. Math. 59, 487 (2006).

[55] M. J. Ablowitz and H. Segur, Solitons and the Inverse Scattering Transform (SIAM, Philadelphia, 1981).

[56] A. Newell, Solitons in Mathematics and Physics (SIAM, Philadelphia, 1985).

[57] J. K. Hunter and J.-M. Vanden-Broeck, J. Fluid Mech. 134, 205 (1983).

[58] J.-M. Vanden-Broeck, Phys. Fluids A 3, 2659 (1991).

[59] J. T. Beale, Commun. Pure Appl. Math. 44, 211 (1991).

[60] S. M. Sun, J. Math. Anal. Appl. 156, 471 (1991).

[61] E. S. Benilov, R. Grimshaw, and E. P. Kuznetsova, Physica D 69, 270 (1993).

[62] R. Grimshaw and N. Joshi, SIAM J. Appl. Math. 55, 124 (1995).

[63] J. P. Boyd, Weakly Nonlinear Solitary Waves and Beyond-AllOrders Asymptotics (Kluwer, Boston, 1998).

[64] E. Lombardi, Oscillatory Integrals and Phenomena Beyond All Algebraic Orders, Lecture Notes in Mathematics Vol. 1741 (Springer-Verlag, Berlin, 2000).

[65] A. R. Champneys, B. A. Malomed, J. Yang, and D. J. Kaup, Physica D 152-153, 340 (2001).

[66] R. Grimshaw and G. Iooss, Math. Comput. Simul. 62, 31 (2003).

[67] C. Fochesato, F. Dias, and R. Grimshaw, Physica D 210, 96 (2005).

[68] V. V. Voronovich, I. A. Sazonov, and V. I. Shrira, J. Fluid Mech. 568, 273 (2006).

[69] G. M. Muslu and H. A. Erbay, Comput. Math. Appl. 45, 503 (2003).

[70] S. D. Griffiths, R. H. J. Grimshaw, and K. R. Khusnutdinova, Physica D 214, 1 (2006).

[71] F. D. Murnaghan, Finite Deformations of an Elastic Solid (Wiley, New York, 1951).

[72] A. I. Lurie, Nonlinear Theory of Elasticity (Elsevier, Amsterdam, 1990). 